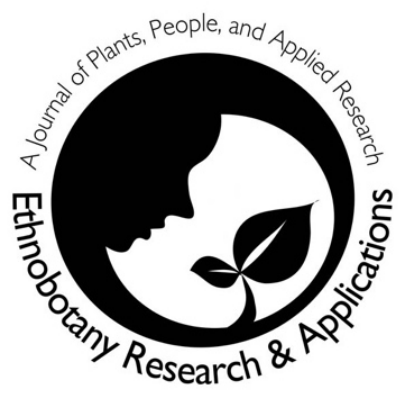

\title{
Influence of Ayurveda in traditional health practice of tribe of Shahapur and Jawhar forest area of Maharashtra
}

Rasika Kolhe, Arun Gurav, Goli P. Prasad, Chinmay Rath, A.K. Mangal and N. Srikanth

\section{Correspondence}

Rasika Kolhe ${ }^{1}$, Arun Gurav ${ }^{1}$, Goli P. Prasad ${ }^{2}$, Chinmay Rath ${ }^{3}$, A.K. Mangal ${ }^{3}$ and N. Srikanth ${ }^{3}$

${ }^{1}$ Regional Ayurveda Research Institute (CCRAS), Kothrud, Pune-411038, Maharashtra-India

${ }^{2}$ National Center of Indian Medical Heritage (CCRAS), Gaddiannaram, Hyderabad- 500036, Telangana-India

${ }^{3}$ Central Council For Research In Ayurvedic Sciences, Janakpuri-110058, New Delhi-India

*Corresponding Author: gurav_am@yahoo.co.in

Ethnobotany Research \& Applications 22:19 (2021)

\section{Research}

\begin{abstract}
Background. The ancient traditional system of medicine of India knows as "Ayurveda" uses natural sources as the base of medicine as per fundamental principles. The traditional folk healers also retain such knowledge gained from their ancestors of proper usage of natural resources available in their native flora, for the management of disease. Hence this study was planned to document tribal knowledge from the Jawhar and Shahapur Forest division of Maharashtra, India and verified with reported information of the Ayurveda.
\end{abstract}

Methods. Data was collected by recording detail interview of 47 respondents during Medico-ethno-botanical survey in the year 2018-19. Collected data was analyzed using quantitative tools, like Use Value (UV), Informant Consensus Factor (ICF), Family Importance Value (FIV), and Fidelity Level (FL). Collected claims were also validated from 20 books including classical textbooks of Ayurveda and ethno medicine and other search engines.

Results: Total 182 Ethno medicinal claims consisting of 10 compound formulations were reported for combating 68 different types of diseases and symptoms. Data of 99 plant species belonging to 88 genera and 48 families were recorded from 47 respondents. The highest use value (UV) 0.13 was recorded for Holarrhena pubescens Wall. ex G. Don and Tinospora cordifolia (Willd.) Miers. It was observed that 16 claims of a single drug and all compound formulations are not recorded in the classical textbooks of Ayurveda

Conclusion. Almost $95 \%$ of tribal health practice is according to the Ayurveda. However, novel folk claims could be explored for bioactive and further pharmacological activities to introduce in Ayurvedic Pharmacopoeia.

Key words: Ayurveda, Ethno medicine, Folklore claim, Validation.

\section{Background}

The human race has long relied on plants for basic necessities such as sustenance and health. Since classical times, natural resources have always been a source of medicines for India's traditional health care systems, such as Ayurveda, Unani, Siddha, Sowa Rigpa (https://main.ayush.gov.in/ayush-systems). As a result of civilization, direct reliance on natural resources has decreased. However, according to WHO, the majority of the world's population 
(80\%) is contingent on natural resources to tackle a variety of diseases (Azaizeh et al. 2003, https://www.who.int/malaria/publications/world_mala ria_report). Ayurveda is a science having its own fundamental principles like prakriti (human body constitution), panchamahabhuta (Five basic elements- Earth, Water, Fire, Air, and Space), tridosha (three regulatory functional factors of the body), etc. However, a lack of exchange of information from Ayurveda to modern science around the world has resulted in the waning of this priceless traditional wisdom (Jaiswal \& Williams 2017).

The traditional folk healers retain such knowledge of the proper usage of natural resources from their native flora for the management of diseases gained from their predecessor and transferred to the next generation.

The documentation of such traditional knowledge is a necessity in the field of drug research to thrive in the modern era (Chaudhary \& Muchtar 2001). As evidenced by the preceding paper, indigenous plant species played vital role in various innovations (Suntar 2019). With this background, it is important to document ethnic knowledge through extensive Medico Ethno-Botanical studies to enrich the health care system. Such documentation and reporting are equally important for the conservation of native flora.

In the Charak Samhita, one of the ancient Ayurvedic classical textbooks, the importance of ethnomedicines is well recognized (Agnivesh 2007) and since then several classical textbooks, notably Nighantu (Lexicon), have been modified by incorporating knowledge shared by tribal (Pandit 2006). In the contemporary period also, substantial research is undertaken in the field of medicinal plants and ethnomedicine, even so, chemical, and pharmacological screening of just $6 \%$ of total plant species might be produced as lifesaving medications (Goswami et al. 2002). To scientifically establish a drug and enrich the science and health care system, the documentation of ethnomedicinal therapeutic use of medicinal plants needed improvisation and accuracy.

The association between the use of medicinal plants and tribal communities is always inspiring but over the period it is observed that dependency of tribal on the natural sources is slowing down due to cultural up-gradation, land degradation, deforestation, and various developmental activities. Approximately 3.5 million tribal people are estimated to have left agriculture and related activities in the last decade (Press Information Bureau. 2021. https://pib.gov.in/). Hence it is high time to codify and preserve such important tribal information by adhering to a code of ethics and conduct. (International Society of Ethnobiology Code of Ethics with 2008 additions) (https://www.ethnobiology.net/ code-of-ethics).

Further, to understand the impact of Ayurveda on tribal health practices, it is necessary to document and validate ethnomedicinal claims and medicinal plants used by tribal folk healers for their mention in the classic Ayurvedic texts. It is also necessary to validate novel claims and include the native flora used by tribal in Ayurvedic compendiums. This study will take one step closer towards merging the Ethnic and Ayurvedic knowledge recorded from the medico-ethnobotanical survey studied in Jawhar and Shahapur forest division of Than forest circle, Maharashtra, India.

The aim of the present study was documentation of the traditional knowledge from the tribal dwelling in the study area and validation of the collected data from the classical literature of Ayurveda to appraise the influence of the traditional Indian system of medicine i.e., Ayurveda on tribal health practices. The scientific presentation of data will help to understand the need for conservation of such knowledge and novel claims collected during the survey would pave the path for further scientific study.

\section{Materials and Methods}

\section{Survey area}

The medico-ethnobotanical survey was conducted in tribal pockets of Shahapur and Jawhar forest division of Thane Forest Circle of Maharashtra, India. (Fig. 1.) The selected area falls under the Northern Western Ghats region, which is, rich in biodiversity with a moist deciduous type of forest ecosystem (http://cgwb.gov.in/District_Profile/Maharashtra/Thane.pdf). Shahapur lies in the Western Ghats located at $19.45^{\circ} \mathrm{N}$; $73.33^{\circ} \mathrm{E}$ and Jawhar lies in between $19.92^{\circ} \mathrm{N} 73.23^{\circ} \mathrm{E}$ (https://en.wikipedia.org). The selected area covers $40.06 \%$ and $96.97 \%$ of the tribal population in the Shahapur and Jawhar area respectively as per the census of 2011 (District Census Handbook Thane. 2011, census. https://censusindia.gov.in/2011,census/). Thane forest circle covers about 3463 square $\mathrm{km}$. the area under the forest which is about $37.10 \%$ of the total geographical area. Out of this, $20.62 \%$ of forest area is in the Shahapur forest division and $9.99 \%$ in the Jawhar forest division (http://www.dcmsme.gov.in/). Shahapur and Jawhar forest divisions are situated merely 120-150 km from the cities 
like Thane and Nashik, still the lifestyle, living standard, occupation, economic status, and culture are entirely different. Jawhar forest division falls under the newly formed Palghar district, which was carved out of Thane district on 1st August 2014, whereas Shahapur forest division comes under the Thane district of Maharashtra, India.

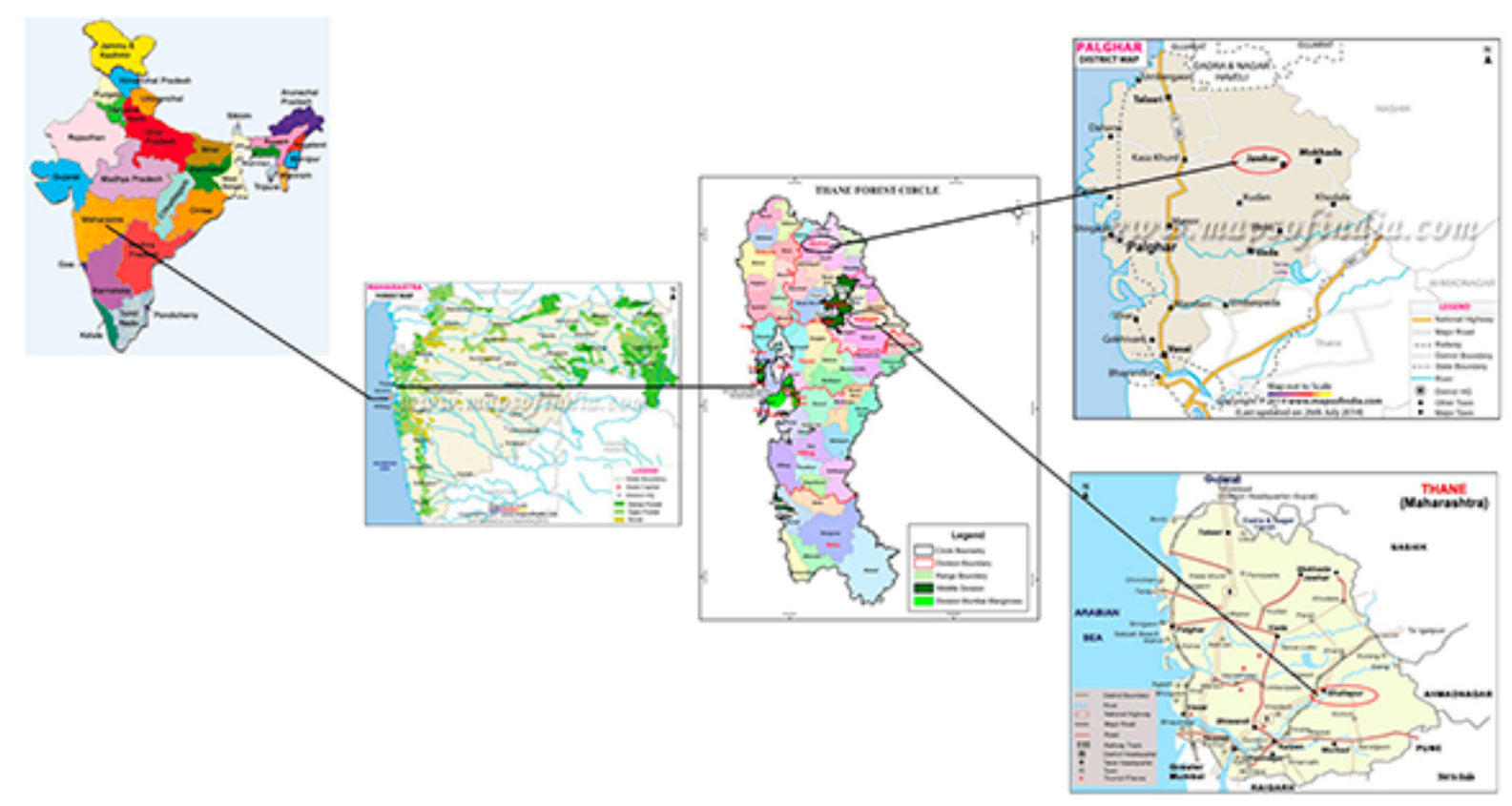

Figure 1. The location of the surveyed area

\section{Weather}

This area has high humidity nearly throughout the year, an oppressive summer season, and well distributed and heavy rainfall during the South-West monsoon season. Overall, there are four seasons summer, monsoon, postmonsoon season, and winter season.

\section{Tribal communities in the study area}

Tribal like katkari, kolams, madiagonds, bhils, gonds, mahadevkolis, malharkolis, and kokans reside in the surveyed areas among which katkari (kathodi), madiagond, and kolam are the vulnerable tribal groups (Major Caste and Tribes.https://gazetteers.maharashtra.gov.in/cultural.maharashtra.gov.in).

\section{Data collection and identification of plant species}

A systematic survey study was planned in each quarter during the year 2018-19. After obtaining permission from the government official representative of the forest department of the Thane forest circle, Maharashtra, India; a survey team consisting of Ayurvedic physicians and botanists visited villages for 10-15 days. A specially designed semi-structured questionnaire was prepared to document ethnomedicinal knowledge about the usage of natural sources for health care. Before conducting the survey, all the participants were explained the clear objective of the study in a language understood by them. Prior informed consent of the local forest department head, village heads, and tribal leaders was also taken whereas a local person of the community well versed in the local language also accompanied as a guide to assist the survey team during the interview for better understanding of local terms used by tribal communities. Interviews of the local vaidu / bhagat (Traditional doctor), folk healers, employees of forest departments like forest guards, watchmen, and elderly people having experience with the usage of medicinal plants were conducted only after obtaining prior informed consent. The information shared by the respondents was filled in the special design LHT (Local Health Tradition) proforma which served as primary data. Information regarding the local plant names, part(s) used, methods of preparation and application, dose, duration, indication, method of diagnosis, etc. along with the details of knowledge providers were recorded during the interview. Moreover, a field survey was also conducted along with the tribal for the collection of plant samples and additional information about their tradition and culture were recorded from local informers by detail formal and informal discussions. 
Taxonomical identification and herbarium:

The medicinal plant species used by the local communities of the study area were collected from the study area and authenticated with the help of flora (Singh \& Karthikeyan 2001), published literature (https://www.teriin.org/projects/), and the taxonomist of the Jawhar College and Botanist of the Institute. Collected Data of medicinal plants was verified for Sanskrit and local names from websites like envis FRLHT (Foundation for Revitalization of Local Health Traditions) (http://envis.frlht.org), biodiversity portal (https://indiabiodiversity.org). The Plant List (http://www.theplantlist.org) and International Plant Name Index (http://www.ipni.org), were used for the correct botanical name. The life form was categorized as herbs, shrubs, climbers, liana, and trees according to the proposed and modified system (Raunkier 1934, Brown 1977) The herbarium of rare and uncommon plant species was prepared and the field book along with voucher specimens was submitted in Herbarium of the Regional Ayurveda Research Institute, Pune, Maharashtra, India.

\section{Data Organization}

The collected ethnobotanical data were entered into Excel spreadsheet 2007 and summarized using graphical statistical methods such as percentages. The part used by the healers for the preparation of ethno medicines was grouped under 15 classes, that is, leaves, whole plant, root, fruit, bark, etc. Human ailments treated in the study area were classified as per the Ayurvedic terminology of diseases; based on the symptoms and disease narrated by respondents. Described route of administration was also categorized as per the Ayurvedic terminology of administration of medicine. The demographic data regarding informants' gender, age groups, educational status, and occupation was also carried out.

\section{Quantitative analysis}

The ethnobotanical data were analyzed using different quantitative indices including Informant Consensus Factor (ICF), Use Value (UV), and Family Importance Value (FIV) and Fidelity level (FL). Data was reported in numbers and percentages.

\section{Informant Consensus Factor (ICF)}

ICF value specifies the informant's consent on the medicinal plant utilization species and evaluates variability in the method of utilization against reported diseases. During calculating ICF values, diseases were broadly categorized into different categories. The maximum ICF value i.e. close to 1 indicates that popular species are employed by a huge number of local populations due to their authenticity regarding diseases. Nevertheless, a low ICF index close to 0 defines that the informants practice this species randomly to treat reported disease conditions. (Canales et al. 2005). ICF was calculated by using formula:

$$
\text { ICF }=\mathrm{Nur}_{\mathrm{ur}}-\mathrm{N}_{\mathrm{t}} / \mathrm{Nur}_{\mathrm{ur}}-1
$$

Where ICF = informants consensus factor, $\mathrm{N}_{\mathrm{ur}}=$ number of use citation, $\mathrm{N}_{\mathrm{t}}=$ number of used species

\section{Use value (UV)}

Use value (UV) determines the relative importance of the uses of plant species. It is calculated using the following formula (Philips \& Gentry 1993)

$$
U V_{i}=\Sigma U_{i} / N
$$

Where $\mathrm{Ui}_{i}$ the number of use reports cited by an informant for a particular plant species and $\mathrm{N}$ is the total number of informants interviewed during the survey.

\section{Family Importance Value (FIV)}

Family Importance Value (FIV) gives the local importance of the families of wild species. It was calculated by calculating the percentage of informants mentioning a specific family. (Vitalini et al. 2013)

$$
\mathrm{FIV}=\mathrm{FC} / \mathrm{N} \times 100
$$

Where $\mathrm{FC}$ is the number of informants mentioning the family and $\mathrm{N}$ is the total number of informants participated in the study. 
Fidelity level (FL)

Fidelity level (FL) is used to calculate the relative frequency of citation (Friedman et al. 1986, Ouedraogo et al. 2019) The percentage of informants who claim to use specific plant species for specific healing processes is referred to as FL. This demonstrates people's preference for a certain plant species in a specific therapeutic therapy. The following formula was used to calculate it:

$F L(\%)=N p / N X 100$

Where $\mathrm{Np}$ is the number of informants who mentioned or claimed the use of plant species for a particular ailment. $\mathrm{N}$ is the total number of informants who cited the plant species for various kinds of medicinal treatment.

\section{Validation of folk knowledge}

After entering the data in LHT (Local Health Tradition) format, the collected folk claims were validated from the classical text of Ayurveda (Agnivesh 2007, Sushrut 2009, Vagbhat 2002), etc., Nighantus (http://niimh.nic.in/ebooks/e-Nighantu/), Glossary of Indian Medicinal Plants (Chopra 1956), Medicinal Plants used in Ayurveda, (Sharma 1998), Classical Uses of Medicinal Plants (Sharma 2004), An Appraisal of Tribal Folk Medicines (Anonymous 1999), The Ethnobotany of Eastern Ghats (Rao \& Henry 1996), Medico Ethnobotany of Utter Pradesh (Uniyal \& Tiwari, 1993), Medico-botanical exploration of Orissa (Hemadri et al. 1996), Ethnobotany (Trivedi 2002), Indian Materia Medica (Nadkarni 1999), Database of Medicinal Plants Vol 1-8 (Sharma et al. 2001), Ayurvedic Pharmacopeia of India (Anonymous API 1999) and Ayurvedic Formulary of India (Anonymous AFI 2000) to identify the novel folk claim or plant with a new indication or new mode of administration. Compiled folk claims were also searched on different search engines like Google Scholar, Pubmed to validate the reported claims. After analysis of the collected data from classical texts and published literature, through reference books and journals, the claims which are not reported in any literature form are considered as novel claims.

\section{Results}

\section{Demographic Data}

The present study recorded data from a total of 47 respondents. Demographic features of the informants showed that, amongst the total respondents $(n=47), 93.61 \%$ were male whereas $6.38 \%$ were female. Amongst the respondent, $38.29 \%$ were folk healers and $61.70 \%$ were informants consisting of watchmen (19.14\%), forest guard (23.40\%), farmers (48.93\%), laborers (12.76\%). Elderly and experienced respondents were (61.70\%) within the 45 70 years age category, whereas youth respondents between the $25-44$ years of age group were $38.29 \%$ In terms of educational status, $66.7 \%$ of the respondents were literate having primary and secondary education; whereas (46.80\%) were illiterates and spoke the local language, i.e. Marathi. The occupational distribution of the respondents shows that $(48.93 \%)$ were farmers, while $(51.06 \%)$ were belonging to the forest department working as a forest guard, watchman, and forest labor. Maximum (85.10\%) respondents have learned by self-interest, whereas $14.89 \%$ of respondents, especially folk healers have learned from their gurus (teacher)/ guide/ parents. Amongst the interviewed individuals, $6.38 \%$ of folk healers had a family history of the tradition of this occupation (Table. 1. )

Table.1. Demographic information of respondents

\begin{tabular}{llll}
\hline Variables & Category & Number & Percentage \\
\hline Gender & & & \\
\hline & Male & 44 & $93.61 \%$ \\
\hline Age group & Female & 03 & $6.38 \%$ \\
\hline & & & \\
\hline Educational status & $45-70$ & 18 & $61.70 \%$ \\
\hline & $25-44$ & 29 & $38.29 \%$ \\
\hline \multicolumn{5}{c}{} & & \\
\hline & Illiterate & 22 & $46.80 \%$ \\
\hline Major occupation & Primary & 15 & $31.91 \%$ \\
\hline & Secondary & 7 & $14.89 \%$ \\
\hline \multicolumn{5}{c}{ Graduates } & 3 & $6.38 \%$ \\
\hline Area of source & & & \\
information & Farming & 23 & $48.93 \%$ \\
\hline & Forest department & 24 & $51.06 \%$ \\
\hline & & & \\
\hline & Self interested & 40 & $85.10 \%$ \\
\hline
\end{tabular}


Table. 2. Medico-ethnobotanical claims of single drug reported during the survey of Jawhar and Shahapur Forest division.

\begin{tabular}{|c|c|c|c|c|c|c|c|c|}
\hline Botanical Name & Local name & Sanskrit name & $\begin{array}{l}\text { Voucher } \\
\text { Specimen } \\
\text { Number }\end{array}$ & Part Used & $\begin{array}{l}\text { Form of } \\
\text { formulations }\end{array}$ & Indication & Dose & $\begin{array}{l}\text { Mode of application and } \\
\text { validation }\end{array}$ \\
\hline $\begin{array}{l}\text { Acacia catechu (L.F.) } \\
\text { Willd. (Leguminosae) }\end{array}$ & khaira & khadira & 1092 & Heartwood & heartwood & $\begin{array}{l}\text { mukhapaka } \\
\text { (Stomatitis / Oral } \\
\text { ulcers) }\end{array}$ & $500 \mathrm{mg}$ & $\begin{array}{l}\text { Masticate the heartwood with } \\
\text { betel leaf (Piper betel L.). Twice } \\
\text { a day for 1-2 days. } \\
\text { (Anonymous 1999) }\end{array}$ \\
\hline $\begin{array}{l}\text { Acacia nilotica (L.) Delile } \\
\text { (Leguminosae) }\end{array}$ & babhali & babhula & 2248 & Stem Bark & churna (powder) & $\begin{array}{l}\text { dantashula } \\
\text { (Toothache) }\end{array}$ & $5 \mathrm{gm}$ & $\begin{array}{l}\text { Take one teaspoonful of dry } \\
\text { stem bark powder in } 1 \text { glass of } \\
\text { water. Gargle once a day for } 3 \\
\text { days. (Ali et al. 2012) }\end{array}$ \\
\hline $\begin{array}{l}\text { Acacia nilotica (L.) Delile } \\
\text { (Leguminosae) }\end{array}$ & babhali & babhula & 2248 & Stem Bark & $\begin{array}{l}\text { gomutrakwadith } \\
\text { a twaka (bark } \\
\text { boiled in cow } \\
\text { urine) }\end{array}$ & $\begin{array}{l}\text { pad daha } \\
\text { (Burning sensation in } \\
\text { sole/ Peripheral } \\
\text { neuropathy) }\end{array}$ & $\begin{array}{l}\text { As required (kept } \\
\text { up to overnight) }\end{array}$ & $\begin{array}{l}\text { Boil stem bark in cow's urine (1 } \\
\text { lit gomutra) till it becomes } \\
\text { smooth. Apply the bark on the } \\
\text { sole, tie with the help of cloth } \\
\text { during nighttime. Repeat it for } \\
3 \text { days. (Sharma 1998) }\end{array}$ \\
\hline $\begin{array}{l}\text { Acacia pennata (L.) Willd. } \\
\text { (Leguminosae) }\end{array}$ & chilar & vallikhadira & 470 & Stem Bark & $\begin{array}{l}\text { swarasa } \\
\text { (juice) }\end{array}$ & $\begin{array}{l}\text { sarpadansha } \\
\text { (Snakebite) -for } \\
\text { animals }\end{array}$ & 1 Litre & $\begin{array}{l}\text { Collect the fresh stem bark and } \\
\text { prepare the juice by crushing } \\
\text { and adding 1lit of water. } \\
\text { Administer orally using a } \\
\text { green colour bottle } \\
\text { immediately after the snake } \\
\text { bite. Root (Kumar \& } \\
\text { Suryanarayana 2011) }\end{array}$ \\
\hline $\begin{array}{l}\text { Achyranthes aspera L. } \\
\text { (Amaranthaceae) }\end{array}$ & aghada & apamarga & 14055 & Root & $\begin{array}{l}\text { kalka } \\
\text { (paste) }\end{array}$ & $\begin{array}{l}\text { vruschik dansha } \\
\text { (scorpionsting) }\end{array}$ & As required & $\begin{array}{l}\text { Prepare the paste by adding } \\
\text { sufficient amount of water. } \\
\text { Apply fine paste locally on the } \\
\text { affected area after the sting. } \\
\text { (Sharma et al. 2001; Vaidya } \\
\text { 1999) }\end{array}$ \\
\hline $\begin{array}{l}\text { Achyranthes aspera } \mathrm{L} . \\
\text { (Amaranthaceae) }\end{array}$ & aghada & apamarga & 14055 & Seed & vati (pills) & $\begin{array}{l}\text { shwana dansha } \\
\text { (dog bite) }\end{array}$ & $10-12$ seeds & $\begin{array}{l}\text { Add a sufficient quantity of } \\
\text { Jaggery to prepare a pill. } \\
\text { Administer orally once. } \\
\text { (Sharma 1998, Vaidya 1999) }\end{array}$ \\
\hline $\begin{array}{l}\text { Achyranthes aspera } \mathrm{L} . \\
\text { (Amaranthaceae) }\end{array}$ & aghada & apamarga & 14055 & Leaf & vati (pill) & $\begin{array}{l}\text { twakgata } \\
\text { khajurkantaka shula } \\
\text { (twakgat shalya } \\
\text { vedana) unexplored } \\
\text { painful Phoenix etc. } \\
\text { Thorns under the skin. }\end{array}$ & 2-3 pills & $\begin{array}{l}\text { Make pills by adding Jaggery } \\
\text { in the paste of leaf and } \\
\text { administer orally twice a day } \\
\text { for one day. (Vaidya 1999) }\end{array}$ \\
\hline $\begin{array}{l}\text { Achyranthes aspera L. } \\
\text { (Amaranthaceae) }\end{array}$ & aghada & apamarga & 14055 & $\begin{array}{l}\text { Whole } \\
\text { Plant }\end{array}$ & kshara (alkali) & $\begin{array}{l}\text { ashmari } \\
\text { (Urolithiasis) }\end{array}$ & $500 \mathrm{mg}$ & $\begin{array}{l}\text { Prepare ash }(2-3 \mathrm{~kg}) \text { of the } \\
\text { whole part of the plant and } \\
\text { soak it overnight, filter the }\end{array}$ \\
\hline
\end{tabular}




\begin{tabular}{|c|c|c|c|c|c|c|c|c|}
\hline & & & & & & & & $\begin{array}{l}\text { supernatant through the cloth, } \\
\text { allow it to settle for } 24 \text { hours, } \\
\text { repeat the same procedure for } \\
4-6 \text { days. Then boil the final } \\
\text { filtrate and evaporate the } \\
\text { filtrate till the alkali (kshara) } \\
\text { deposition occurs. Administer } \\
\text { orally twice a day for } 3 \text { days. } \\
\text { (Desai } 2015 \text {, Bhavmishra 2006) }\end{array}$ \\
\hline $\begin{array}{l}\text { Haldina cordifolia (Roxb.) } \\
\text { Ridsdale (Rubiaceae) }\end{array}$ & $\begin{array}{l}\text { haidu } \\
\text { (bhunga } \\
\text { powder) }\end{array}$ & haridru & 14176 & $\begin{array}{l}\text { Heart } \\
\text { wood }\end{array}$ & $\begin{array}{l}\text { siddha } \\
\text { taila (medicated } \\
\text { oil) }\end{array}$ & $\begin{array}{l}\text { dadru/ twakvikara } \\
\text { (ring worm infection) }\end{array}$ & As required & $\begin{array}{l}\text { Boil small cut pieces of heart } \\
\text { wood in soya bean oil till the } \\
\text { essence of heart wood comes } \\
\text { into the oil and the pieces } \\
\text { become black. Filter it, keep it } \\
\text { in airtight bottle. Apply } \\
\text { externally whenever needed. } \\
\text { (Natarajan et al. 2000, Vaidya } \\
\text { 1999) }\end{array}$ \\
\hline $\begin{array}{l}\text { Aegle marmelos (L.) } \\
\text { Corrêa (Rutaceae) }\end{array}$ & bel & bilwa & 3145 & Leaf & $\begin{array}{l}\text { swarasa } \\
\text { (juice) }\end{array}$ & $\begin{array}{l}\text { balaatisara (diarrhoea } \\
\text { in children), amatisara } \\
\text { (diarrhoea with mucus) }\end{array}$ & $1-3$ leaves & $\begin{array}{l}\text { Administer fresh juice orally } \\
\text { once a day for three days on } \\
\text { empty stomach. (Sharma et al. } \\
\text { 2001) }\end{array}$ \\
\hline $\begin{array}{l}\text { Albizia lebbeck (L.) Benth. } \\
\text { (Leguminosae) }\end{array}$ & shirasa & shirish & 664 & Stem Bark & $\begin{array}{l}\text { kwatha } \\
\text { (decoction) }\end{array}$ & $\begin{array}{l}\text { raktarsha (bleeding } \\
\text { piles) }\end{array}$ & $30 \mathrm{ml}$ & $\begin{array}{l}\text { Add one teaspoonful powder } \\
\text { in } 60 \mathrm{ml} \text { of water and boil it on } \\
\text { low flame till it becomes half } \\
\text { quantity. Administer orally } \\
\text { twice a day on an empty } \\
\text { stomach for } 8 \text { days. (Vaidya } \\
\text { 1999) }\end{array}$ \\
\hline $\begin{array}{l}\text { Aloe vera (L.) Burm. f. } \\
\text { (Xanthorrhoeaceae) }\end{array}$ & korphad & kumari & 2250 & Leaf & leaf pulp & visarpa (Herpes) & As required & $\begin{array}{l}\text { Apply leaf pulp locally over the } \\
\text { affected area once in a day for } \\
5 \text { days. (Nadkarni 1999) }\end{array}$ \\
\hline $\begin{array}{l}\text { Amorphophallus } \\
\text { paeoniifolius (Dennst.) } \\
\text { Nicolson (Araceae) }\end{array}$ & lotha & suran & 14601 & Tuber & vati (pills) & $\begin{array}{l}\text { arsha (Piles / } \\
\text { Haemorrhoids) }\end{array}$ & $1-2 \mathrm{gm}$ & $\begin{array}{l}\text { Prepare slices of tuber and dry } \\
\text { it. Prepare coarse powder of } \\
\text { one slice. Coat it with } 5 \mathrm{gm} \\
\text { jaggery administer orally on } \\
\text { empty stomach on alternate } \\
\text { days. Administer } 5 \text { doses. (Dey } \\
\text { et al. 2016) }\end{array}$ \\
\hline $\begin{array}{l}\text { Anacardium occidentale } \\
\text { L. (Anacardiaceae) }\end{array}$ & kaju & kajutak & 14261 & Stem Bark & kalka (paste) & $\begin{array}{l}\text { vrishchikdansha } \\
\text { (scorpion sting) }\end{array}$ & As required & $\begin{array}{l}\text { Without touching peel, a piece } \\
\text { of stem bark is to be removed } \\
\text { with teeth, masticate and } \\
\text { apply on the affected area } \\
\text { immediately. (Patil 2006) }\end{array}$ \\
\hline $\begin{array}{l}\text { Anogeissus latifolia } \\
\text { (Roxb. ex DC.) Wall. ex }\end{array}$ & dhavada & dhav & 14175 & Seed & churna (powder) & $\begin{array}{l}\text { atisara } \\
\text { (diarrhoea) }\end{array}$ & $\begin{array}{l}\text { 3-5 gm seed } \\
\text { powder }\end{array}$ & $\begin{array}{l}\text { Add powder in } 500 \mathrm{ml} \text { water } \\
\text { and administer orally, once a }\end{array}$ \\
\hline
\end{tabular}


Ethnobotany Research and Applications

\begin{tabular}{|c|c|c|c|c|c|c|c|c|}
\hline $\begin{array}{l}\text { Guillem. \& Perr. } \\
\text { (Combretaceae) }\end{array}$ & & & & & & & & $\begin{array}{l}\text { day for } 3 \text { days, in the morning. } \\
\text { (Vaidya 1999) }\end{array}$ \\
\hline $\begin{array}{l}\text { Asparagus racemosus } \\
\text { Willd (Asparagaceae) }\end{array}$ & narak & shatavari & 2352 & Root & root & kamala (Jaundice) & As required & $\begin{array}{l}\text { Prepare } 7 \text { pearl shape beads } \\
\text { from ( } 1 \text { inch/ } 2-3 \text { gm) root and } \\
\text { make a necklace like structure. } \\
\text { Wear this medicated necklace } \\
\text { for } 7 \text { days. }\end{array}$ \\
\hline $\begin{array}{l}\text { Asparagus racemosus } \\
\text { Willd (Asparagaceae) }\end{array}$ & narak & shatavari & 2352 & Root & churna (powder) & $\begin{array}{l}\text { Stanyakhyaya } \\
\text { (Hypogalactia)/ vrikkaj } \\
\text { vikara (kidney } \\
\text { diseases) } \\
\end{array}$ & $5 \mathrm{gm}$ & $\begin{array}{l}\text { Administer powder orally with } \\
\text { a glass of milk, once a day at } \\
\text { night for } 4-5 \text { days. (Sharma et } \\
\text { al. 2001) }\end{array}$ \\
\hline $\begin{array}{l}\text { Azadirachta indica A. } \\
\text { Juss. (Meliaceae) }\end{array}$ & nimba & nimba & 4219 & Leaf & leaves & $\begin{array}{l}\text { kachhu } \\
\text { (scabies) }\end{array}$ & As required & $\begin{array}{l}\text { Take freshly collected leaves } \\
\text { and boil it in water and use } \\
\text { this for a bath. Once a day for } \\
2-3 \text { days. (Sharma 2004) }\end{array}$ \\
\hline $\begin{array}{l}\text { Baliospermum } \\
\text { montanum (J.Burm.) } \\
\text { Suresh (Euphorbiaceae) }\end{array}$ & jivita & danti & 4096 & Latex & latex & $\begin{array}{l}\text { netravikar (Eye } \\
\text { disease/ blindness) }\end{array}$ & $1-2$ drops & $\begin{array}{l}\text { Instil 1-2 drops latex in eyes } \\
\text { once. } \\
\text { Note: the patient will } \\
\text { experience blindness for 2-3 } \\
\text { hours after which the vision } \\
\text { will be clear. (Vaidya 1999) }\end{array}$ \\
\hline $\begin{array}{l}\text { Bambusa arundinacea } \\
\text { Willd. (Poaceae) }\end{array}$ & bambu & vansha & 14356 & Stem bark & vati (pills) & $\begin{array}{l}\text { nalgutha (Marathi) } \\
\text { (umbilical hernia/ } \\
\text { lateral hernia/ } \\
\text { intestinal hernia) }\end{array}$ & $500 \mathrm{mg}-1 \mathrm{gm}$ & $\begin{array}{l}\text { Collect fresh stem bark of } \\
\text { bamboo and add a sufficient } \\
\text { quantity of jaggery and make } \\
\text { pea-sized pills. Administer } \\
\text { orally once a day on an empty } \\
\text { stomach for } 5 \text { days. (Lavekar et } \\
\text { al.2007) }\end{array}$ \\
\hline $\begin{array}{l}\text { Bauhinia racemosa Lam. } \\
\text { (Leguminosae) }\end{array}$ & apata & asmantaka & 14618 & Stem Bark & kalka (paste) & $\begin{array}{l}\text { raktapradar (vaginal } \\
\text { bleeding/metrorrhagia) } \\
\text {; vandhyatwa (female } \\
\text { infertility) }\end{array}$ & $5 \mathrm{gm}$ in $30 \mathrm{ml}$ milk & $\begin{array}{l}\text { Prepare the paste of freshly } \\
\text { collected stem bark scrubbing } \\
\text { on the stone by adding a little } \\
\text { amount of milk. Administer } \\
\text { orally with milk twice a day on } \\
\text { an empty stomach for } 3 \text { days. } \\
\text { (Sharma 1998) }\end{array}$ \\
\hline $\begin{array}{l}\text { Bombax ceiba L. } \\
\text { (Bombacaceae) }\end{array}$ & kate savar & shalmali & 14054 & Flower & kalka (paste) & $\begin{array}{l}\text { arsha (Piles/ } \\
\text { Haemorrhoids) }\end{array}$ & As required & $\begin{array}{l}\text { Prepare the paste of fresh } \\
\text { flowers by crushing or prepare } \\
\text { the paste by adding water to } \\
\text { the powder of dry flowers. } \\
\text { Apply externally twice a day } \\
\text { for two days on the affected } \\
\text { part. (Desai 2015) }\end{array}$ \\
\hline $\begin{array}{l}\text { Butea monosperma } \\
\text { (Lam.) Taub. } \\
\text { (Leguminosae) }\end{array}$ & palas & palash & 14187 & Flower & $\begin{array}{l}\text { hima } \\
\text { (cold infusion) }\end{array}$ & $\begin{array}{l}\text { aniyamit artava } \\
\text { chakra (Irregular } \\
\text { menstrual cycle) }\end{array}$ & $100 \mathrm{ml}$ & $\begin{array}{l}\text { Soak } 10-12 \text { flowers in a glass } \\
\text { of }(100 \mathrm{ml}) \text { water for overnight } \\
\text { and squeeze it, and filter it on } \\
\text { the next day morning. } \\
\text { Administer the filtrate orally }\end{array}$ \\
\hline
\end{tabular}




\begin{tabular}{|c|c|c|c|c|c|c|c|c|}
\hline & & & & & & & & $\begin{array}{l}\text { on empty stomach for } 2-3 \\
\text { days before the menstrual } \\
\text { cycle. (Nadkarni 1999, Vaidya } \\
\text { 1999) }\end{array}$ \\
\hline $\begin{array}{l}\text { Butea monosperma } \\
\text { (Lam.) Taub. } \\
\text { (Leguminosae) }\end{array}$ & palas & palash & 14187 & Flower & churna (powder) & $\begin{array}{l}\text { adhmana (bloating } \\
\text { with distension), } \\
\text { raktapradar } \\
\text { (Metrorrhagia) }\end{array}$ & $10 \mathrm{gm}$ & $\begin{array}{l}\text { Administered orally with water. } \\
\text { (Nadkarni 1999) }\end{array}$ \\
\hline $\begin{array}{l}\text { Butea monosperma } \\
\text { (Lam.) Taub. } \\
\text { (Leguminosae) }\end{array}$ & palas & palash & 14187 & Root & $\begin{array}{l}\text { kalka } \\
\text { (paste) }\end{array}$ & $\begin{array}{l}\text { vruschik dansha } \\
\text { (scorpion sting) }\end{array}$ & As required & $\begin{array}{l}\text { Apply the paste, locally on the } \\
\text { affected area (Vaidya 1999) }\end{array}$ \\
\hline $\begin{array}{l}\text { Calotropis procera (Aiton) } \\
\text { Dryand. (Apocynaceae) }\end{array}$ & rui & arka & 14105 & Stem & dried stem & $\begin{array}{l}\text { ardhavabhedaka } \\
\text { (Migraine) }\end{array}$ & As required & $\begin{array}{l}\text { Make a cigar of dried stem } \\
\text { pieces, light it on one end and } \\
\text { inhale the smoke through } \\
\text { nostrils for 2-3 days. (Sree et } \\
\text { al. 2011) }\end{array}$ \\
\hline $\begin{array}{l}\text { Calotropis procera (Aiton) } \\
\text { Dryand. (Apocynaceae) }\end{array}$ & rui & arka & 14105 & Leaf & leaf coated by oil & $\begin{array}{l}\text { sandhivata (Osteo } \\
\text { arthritis) }\end{array}$ & As required & $\begin{array}{l}\text { Apply mustard oil/edible oil } \\
\text { over the leaves and heat it } \\
\text { slightly, keep it on the affected } \\
\text { area and tie with a cloth. } \\
\text { Repeat the same twice a day } \\
\text { for } 30 \text { days. (Vaidya 1999, } \\
\text { Anonymous 1999) }\end{array}$ \\
\hline $\begin{array}{l}\text { Calotropis procera (Aiton) } \\
\text { Dryand. (Apocynaceae) }\end{array}$ & rui & arka & 14105 & Latex & $\begin{array}{l}\text { vati } \\
\text { (pills) }\end{array}$ & $\begin{array}{l}\text { tamak shwas } \\
\text { (Bronchial asthma) }\end{array}$ & $500 \mathrm{mg}$ & $\begin{array}{l}\text { Collect the latex before sunrise } \\
\text { and add a sufficient amount of } \\
\text { besan (Chickpea flour) and } \\
\text { make small pills (Chickpea } \\
\text { size). Administer internally } \\
\text { with water twice a day for } 3 \\
\text { days. . (Vaidya 1999) }\end{array}$ \\
\hline $\begin{array}{l}\text { Calycopteris floribunda } \\
\text { (Roxb.) Lam. ex Poir. } \\
\text { (Combretaceae) }\end{array}$ & ukasa & pullani & 14330 & $\begin{array}{l}\text { Tender } \\
\text { Leaf }\end{array}$ & tender leaf & $\begin{array}{l}\text { udarshula } \\
\text { (abdominal colic) }\end{array}$ & $2-3$ tender leaves & $\begin{array}{l}\text { Masticate 2-3 tender leaves } \\
\text { whenever required. (Nadkarni } \\
\text { 1999, Desai 2015, Anonymous } \\
\text { 1999) }\end{array}$ \\
\hline $\begin{array}{l}\text { Calycopteris floribunda } \\
\text { (Roxb.) Lam. ex Poir. } \\
\text { (Combretaceae) }\end{array}$ & ukasa & pullani & 14330 & $\begin{array}{l}\text { Tender } \\
\text { Leaf }\end{array}$ & swarasa (juice) & $\begin{array}{l}\text { shwandansha } \\
\text { (dog bite) }\end{array}$ & $30 \mathrm{ml}$ & $\begin{array}{l}\text { Administer juice orally once a } \\
\text { day (Upadhye et al. 1986) }\end{array}$ \\
\hline $\begin{array}{l}\text { Capparis spinosa } \mathrm{L} . \\
\text { (Capparaceae) }\end{array}$ & waghoti & himsra & 14245 & Root & kalka (paste) & $\begin{array}{l}\text { arsha } \\
\text { (piles/haemorrhoids) }\end{array}$ & As required & $\begin{array}{l}\text { Apply paste locally on the } \\
\text { affected area at bedtime and } \\
\text { keep overnight for } 7 \text { days. } \\
\text { (Anonymous 1999) }\end{array}$ \\
\hline $\begin{array}{l}\text { Careya arborea Roxb. } \\
\text { (Lecythidaceae) }\end{array}$ & kumbhi & kumbhi & 14337 & Stem Bark & kalka (paste) & $\begin{array}{l}\text { sandhivata (osteo } \\
\text { arthritis) }\end{array}$ & As required & $\begin{array}{l}\text { Apply paste locally over the } \\
\text { affected area twice a day for } \\
\text { two days. (Vaidya 1999) }\end{array}$ \\
\hline $\begin{array}{l}\text { Careya arborea Roxb. } \\
\text { (Lecythidaceae) }\end{array}$ & kumbhi & kumbhi & 14337 & Stem Bark & $\begin{array}{l}\text { swarasa } \\
\text { (juice) }\end{array}$ & $\begin{array}{l}\text { udarshula } \\
\text { (abdominal colic }\end{array}$ & $50 \mathrm{~m}$ & $\begin{array}{l}\text { Soak } 2-3 \text { inch piece in half } \\
\text { glass }(50 \mathrm{ml}) \text { water and filter it }\end{array}$ \\
\hline
\end{tabular}




\begin{tabular}{|c|c|c|c|c|c|c|c|c|}
\hline & & & & & & & & $\begin{array}{l}\text { and drink twice a day, on } \\
\text { empty stomach in the morning } \\
\text { (Nadkarni 1999) }\end{array}$ \\
\hline $\begin{array}{l}\text { Carissa carandas } \mathrm{L} . \\
\text { (Apocynaceae) }\end{array}$ & karvanda & karamarda & 14117 & Root & root & $\begin{array}{l}\text { dantashula (dental } \\
\text { carries) }\end{array}$ & $1-2$ inch & $\begin{array}{l}\text { Masticate and hold } 1-2 \text { inch } \\
\text { piece of the root between } \\
\text { aching tooth for } 10 \text { minutes, } \\
\text { once a day for } 2-3 \text { days. } \\
\text { (Kaunda et al. 2017) }\end{array}$ \\
\hline $\begin{array}{l}\text { Casearia graveolens } \\
\text { Dalzell (Salicaceae) }\end{array}$ & kirmir & chilhaka & 14312 & Twig & twig & $\begin{array}{l}\text { sarpadansha (snake } \\
\text { bite) }\end{array}$ & As required & $\begin{array}{l}\text { Hold a } 2-3 \text { inch piece of a } \\
\text { tender twig in hand } \\
\text { immediately after the snake } \\
\text { bite for } 2-3 \text { hours to control } \\
\text { vishavega (spreading of } \\
\text { poison) till further proper } \\
\text { medical treatment is initiated. } \\
\text { (Lele et al. 2017) }\end{array}$ \\
\hline $\begin{array}{l}\text { Cassia fistula } \mathrm{L} \text {. } \\
\text { (Leguminosae) }\end{array}$ & bahava & aragvadha & 14154 & Stem Bark & $\begin{array}{l}\text { kalka (paste) and } \\
\text { kwatha } \\
\text { (decoction) }\end{array}$ & visarpa (herpes) & $\begin{array}{l}1-2 \text { inch piece and } \\
30 \mathrm{ml} \text { kwatha }\end{array}$ & $\begin{array}{l}\text { Rub stem bark over a stone } \\
\text { and apply the paste over the } \\
\text { affected area; prepare a } \\
\text { decoction by boiling the root } \\
\text { in } 60 \mathrm{ml} \text { water and reduce it to } \\
\text { half quantity. Administered } \\
\text { orally on stomach for } 5 \text { days } \\
\text { (Nadkarni 1999) }\end{array}$ \\
\hline $\begin{array}{l}\text { Cassia fistula L. } \\
\text { (Leguminosae) }\end{array}$ & bahava & aragvadha & 14154 & Leaf & kalka (paste) & $\begin{array}{l}\text { dadru } \\
\text { (ringworm infection) }\end{array}$ & $\begin{array}{l}\text { 3-4 fresh tender } \\
\text { leaves }\end{array}$ & $\begin{array}{l}\text { Prepare a paste by crushing 3- } \\
4 \text { fresh tender leaves and rub } \\
\text { over the affected area once a } \\
\text { day, for 2-3 days. (Vaidya 1999; } \\
\text { Agnivesh 2007) }\end{array}$ \\
\hline $\begin{array}{l}\text { Cassia fistula } \mathrm{L} . \\
\text { (Leguminosae) }\end{array}$ & bahava & aragvadha & 14154 & Pod & $\begin{array}{l}\text { swarasa } \\
\text { (juice) }\end{array}$ & $\begin{array}{l}\text { mutravikar - } \\
\text { mutrakrucchra } \\
\text { (Painful micturation) } \\
\end{array}$ & $5 \mathrm{ml}$ & $\begin{array}{l}\text { Administer orally once a day } \\
\text { for three days on an empty } \\
\text { stomach. (Deshpande 2018) }\end{array}$ \\
\hline $\begin{array}{l}\text { Catunaregam spinosa } \\
\text { (Thunb.) Tirveng. } \\
\text { (Rubiaceae) }\end{array}$ & galphal & madanphal & 4289 & Seed & kalka (paste) & $\begin{array}{l}\text { pashanagardhabha } \\
\text { (Mumps) }\end{array}$ & As required & $\begin{array}{l}\text { Prepare the paste by rubbing } \\
\text { ripe fruit and apply locally, } \\
\text { keep overnight, once in a day, } \\
\text { for } 3 \text { days. (Anonymous 1999, } \\
\text { Vaidya 1999) }\end{array}$ \\
\hline $\begin{array}{l}\text { Celosia argentea L. } \\
\text { (Amaranthaceae) }\end{array}$ & kurdu & mayurshikha & 14622 & $\begin{array}{l}\text { Root and } \\
\text { Seed }\end{array}$ & churna (powder) & $\begin{array}{l}\text { mutrashmari } \\
\text { (urolithiasis) }\end{array}$ & $5 \mathrm{~g} / 5 \mathrm{ml}$ & $\begin{array}{l}\text { Administer } 5 \text { gm powder orally } \\
\text { twice a day with water for } \\
\text { three days on empty stomach. } \\
\text { (Desai 2015) }\end{array}$ \\
\hline $\begin{array}{l}\text { Cissampelos pareira L. } \\
\text { (Menispermaceae) }\end{array}$ & tanvel & patha & 14016 & Leaf & swarasa (juice) & atisara (diarrhea) & $5-10 \mathrm{ml}$ & $\begin{array}{l}\text { Administer orally once in a } \\
\text { day. (Bhavmishra 2006) }\end{array}$ \\
\hline $\begin{array}{l}\text { Cissampelos pareira } \mathrm{L} . \\
\text { (Menispermaceae) }\end{array}$ & tanvel & patha & 14016 & $\begin{array}{l}\text { Whole } \\
\text { plant }\end{array}$ & aerial part & $\begin{array}{l}\text { amatisara (stools with } \\
\text { mucus) }\end{array}$ & As require & $\begin{array}{l}\text { Tie stem and aerial part over } \\
\text { the wrist of both hands as }\end{array}$ \\
\hline
\end{tabular}


Ethnobotany Research and Applications

\begin{tabular}{|c|c|c|c|c|c|c|c|c|}
\hline & & & & & & & & $\begin{array}{l}\text { bracelets for 2-3 days. } \\
\text { (Bhavmishra 2006) }\end{array}$ \\
\hline $\begin{array}{l}\text { Clerodendrum serratum } \\
\text { (L.) Moon (Lamiaceae) }\end{array}$ & bharangi & bharangi & 14108 & Leaf & leaf & $\begin{array}{l}\text { galashotha (Throat } \\
\text { Infection) }\end{array}$ & $4-5$ & $\begin{array}{l}\text { Consume freshly collected } \\
\text { tender leaves 3-4 times a day } \\
\text { for 2-3 days. (Sharma 2013) }\end{array}$ \\
\hline $\begin{array}{l}\text { Coriandrum sativum } \mathrm{L} . \\
\text { (Apiaceae) }\end{array}$ & dhane & dhanyak & 1121 & Seed & $\begin{array}{l}\text { hima (cold } \\
\text { decoction) }\end{array}$ & $\begin{array}{l}\text { mutrashmari } \\
\text { (urolithiasis/ renal } \\
\text { calculi) }\end{array}$ & $200 \mathrm{ml}$ & $\begin{array}{l}\text { Crush approx } 30 \mathrm{gm} \text { of seeds } \\
\text { and soak in. } 200 \mathrm{ml} \text { of water } \\
\text { overnight filter and } \\
\text { administered internally the } \\
\text { next morning on empty } \\
\text { stomach. Repeat the same for } \\
7-8 \text { days. (Sharma et al.2001, } \\
\text { Bhavmishra 2006) }\end{array}$ \\
\hline $\begin{array}{l}\text { Costus speciosus (J. } \\
\text { Koenig) Sm. (Costaceae) }\end{array}$ & peva & kebuka & 14035 & Rhizome & swarasa (juice) & $\begin{array}{l}\text { arsha (piles/ } \\
\text { haemorhiods) }\end{array}$ & 1-2 inch piece & $\begin{array}{l}\text { Administer internally with } \\
\text { Jaggery once a day, on empty } \\
\text { stomach, for } 5 \text { days. } \\
\text { (Anonymous 1999) }\end{array}$ \\
\hline $\begin{array}{l}\text { Crotalaria juncea L. } \\
\text { (Leguminosae). }\end{array}$ & zunzuni & shanapushpi & 14248 & $\begin{array}{l}\text { Exocarp of } \\
\text { Fruit }\end{array}$ & churna (powder) & $\begin{array}{l}\text { kasa } \\
\text { (Cough) }\end{array}$ & $5 \mathrm{gm}$ & $\begin{array}{l}\text { Prepare fine powder of 5-6 } \\
\text { exocarps of fruits of Crotolaria } \\
\text { and fill it in the dried leaf of } \\
\text { ashmantak to prepare a cigar. } \\
\text { Light it on one side and inhale } \\
\text { the smoke as and when } \\
\text { required. (Agnivesh 2007) }\end{array}$ \\
\hline $\begin{array}{l}\text { Curculigo orchioides } \\
\text { Gaertn. (Hypoxidaceae) }\end{array}$ & $\begin{array}{l}\text { kali musali } \\
\text { /dhasada }\end{array}$ & mushali & 14616 & Root & root & $\begin{array}{l}\text { shukrakshyaya } \\
\text { (oligospermia) }\end{array}$ & 1-inch piece & $\begin{array}{l}\text { Roast } 1 \text { inch long piece of } \\
\text { fresh/dried root. Administer } \\
\text { orally as required. (Vaidya } \\
\text { 1999; Sharma 2004) }\end{array}$ \\
\hline $\begin{array}{l}\text { Curculigo orchioides } \\
\text { Gaertn. (Hypoxidaceae) }\end{array}$ & $\begin{array}{l}\text { kali musali } \\
\text { /dhasada }\end{array}$ & mushali & 14616 & Root & root & $\begin{array}{l}\text { ashmari } \\
\text { (urolithiasis/ urinary } \\
\text { calculi) }\end{array}$ & 2-3 piece & $\begin{array}{l}\text { Take } 1 / 2 \text { inch root (3-4 Nos.) } \\
\text { and masticate and swallow on } \\
\text { empty stomach and drink } 1 \\
\text { glass of water for } 2 \text { days. } \\
\text { Note: if it is difficult to swallow } \\
\text { the roots, few chanas } \\
\text { (chickpea) can be consumed } \\
\text { along with it. (Bhavmishra } \\
\text { 2006) }\end{array}$ \\
\hline $\begin{array}{l}\text { Curcuma amada Roxb. } \\
\text { (Zingiberaceae) }\end{array}$ & ambehalad & $\begin{array}{l}\text { aamragandhi } \\
\text { haridra }\end{array}$ & 14608 & Rhizome & kalka (paste) & $\begin{array}{l}\text { aghataj shotha, } \\
\text { abhighataj shotha } \\
\text { (traumatic swelling) }\end{array}$ & $3 \mathrm{gm}$-Oral, Local & $\begin{array}{l}\text { Apply the fine paste on the } \\
\text { affected area once a day for } 3 \\
\text { days and administer orally } 3 \\
\text { gm powder (Nadkarni 1999, } \\
\text { Vaidya 1999) }\end{array}$ \\
\hline $\begin{array}{l}\text { Curcuma caesia Roxb. } \\
\text { (Zingiberaceae) }\end{array}$ & kali halad & - & 14876 & Rhizome & rhizome & granthi (cyst) & 1 inch & $\begin{array}{l}\text { Masticate once in a day for } 8 \\
\text { days. (Vaidya 1999. Nadkarni } \\
\text { 1999) }\end{array}$ \\
\hline
\end{tabular}


Ethnobotany Research and Applications

\begin{tabular}{|c|c|c|c|c|c|c|c|c|}
\hline $\begin{array}{l}\text { Curcuma longa L. } \\
\text { (Zingiberaceae) }\end{array}$ & halad & haridra & 2816 & Rhizome & vati (pills) & pandu (anaemia) & $\begin{array}{l}1 \text { pill ( } 2 \mathrm{gm} \text { of } \\
\text { turmeric powder) }\end{array}$ & $\begin{array}{l}\text { Prepare the pills by mixing } \\
\text { turmeric powder and Jaggery } \\
\text { in equal quantity. Administer } \\
\text { orally once in a day on empty } \\
\text { stomach for } 2-3 \text { days. } \\
\text { (Nadkarni 1999) }\end{array}$ \\
\hline $\begin{array}{l}\text { Cyclea peltata (Lam.) } \\
\text { Hook.f. \& Thomson } \\
\text { (Menispermaceae) }\end{array}$ & tanvel & rajpatha & 14630 & $\begin{array}{l}\text { Root and } \\
\text { Leaf }\end{array}$ & $\begin{array}{l}\text { swarasa } \\
\text { (juice) }\end{array}$ & $\begin{array}{l}\text { bala atisara (Diarrhoea } \\
\text { in children) }\end{array}$ & $3-5 \mathrm{ml}$ & $\begin{array}{l}\text { Administer orally once a day } \\
\text { for 3-4days. Also, tie fresh root } \\
\text { around both wrists as a } \\
\text { bangle. (Sharma 1998, } \\
\text { Bhavmishra 2006) }\end{array}$ \\
\hline $\begin{array}{l}\text { Cyclea peltata (Lam.) } \\
\text { Hook.f. \& Thomson } \\
\text { (Menispermaceae) }\end{array}$ & tanvel & rajpatha & 14630 & Stem & stem & atisara (Diarrhoea) & As required & $\begin{array}{l}\text { Tie stems around the wrists of } \\
\text { both hands as a bracelet for 1- } \\
2 \text { days. (Bhavmishra 2006, } \\
\text { Sharma 1998) }\end{array}$ \\
\hline $\begin{array}{l}\text { Cynodon dactylon (L.) } \\
\text { Pers. (Poaceae) }\end{array}$ & harali & durva & 2301 & $\begin{array}{l}\text { Whole } \\
\text { Plant }\end{array}$ & whole part & kamala (Jaundice) & As required & $\begin{array}{l}\text { Keep Brass plate (kansya/ } \\
\text { pital plate) on the head of the } \\
\text { patient and pour water in it, } \\
\text { later add mustard oil in lt. } \\
\text { Then take a bunch of durva } \\
\text { and mix mustard oil and water } \\
\text { by stirring until it becomes } \\
\text { yellow. Repeat the procedure } \\
\text { for 2-3 days. (Billore et al. } \\
2004 \text { ) }\end{array}$ \\
\hline $\begin{array}{l}\text { Cyperus rotundus } \mathrm{L} . \\
\text { (Cyperaceae) }\end{array}$ & lavhal & musta & 4314 & Tuber & tuberous roots. & $\begin{array}{l}\text { amlapitta (Hyper } \\
\text { acidity) }\end{array}$ & $3 g m$ & $\begin{array}{l}\text { Masticate 2-3 gm once a day. } \\
\text { (Nadkarni 1999) }\end{array}$ \\
\hline $\begin{array}{l}\text { Cyperus scariosus R.Br. } \\
\text { (Cyperaceae) }\end{array}$ & nagarmotha & chakranksha & 14877 & Tuber & tailam (oil) & $\begin{array}{l}\text { khalitya } \\
\text { (loss of hair) }\end{array}$ & As required & $\begin{array}{l}\text { In } 250 \text { gm coconut or sesame } \\
\text { oil adds } 50 \text { gm powder and } \\
\text { apply locally on the scalp. } \\
\text { Once a day at night, for 6-10 } \\
\text { months. ( Anonymous. 1999) }\end{array}$ \\
\hline $\begin{array}{l}\text { Dalbergia sissoo DC. } \\
\text { (Leguminosae) }\end{array}$ & shisam & sinsapa & 4221 & Leaf & swarasa (juice) & $\begin{array}{l}\text { mutrashmari } \\
\text { (urolithiasis/ renal } \\
\text { calculi) }\end{array}$ & $2-3$ fresh leaves & $\begin{array}{l}\text { Eat 2-3 fresh leaves twice or } \\
\text { thrice a day for } 15 \text { days. } \\
\text { (Sharma et al. 2001, } \\
\text { Bhavmishra 2006) }\end{array}$ \\
\hline $\begin{array}{l}\text { Dalbergia volubilis Roxb. } \\
\text { (Leguminosae) }\end{array}$ & yelvas & sirisika & 14332 & Stem Bark & fresh stem & $\begin{array}{l}\text { mukhapaka } \\
\text { (mouth ulcer) }\end{array}$ & $5 \mathrm{ml}$ & $\begin{array}{l}\text { Masticate fresh stem bark once } \\
\text { a day, before the meal. } \\
\text { (Sharma 2004) }\end{array}$ \\
\hline $\begin{array}{l}\text { Derris scandens (Roxb.) } \\
\text { Benth. (Leguminosae) }\end{array}$ & $\begin{array}{l}\text { karanjvela } \\
\text { /velikaranja/ } \\
\text { velyakaranj }\end{array}$ & - & 14640 & Root & $\begin{array}{l}\text { dravana } \\
\text { (solution) }\end{array}$ & $\begin{array}{l}\text { stanyakshyaya } \\
\text { (Hypogalactia) }\end{array}$ & $30 \mathrm{ml}$ & $\begin{array}{l}\text { Add 3-5 gm powder in } 500 \mathrm{ml} \\
\text { water, leave it for some time } \\
\text { and filter. Administer the } \\
\text { filtrate orally twice a day for } 3 \\
\text { days. (Natarajan et al.2000) }\end{array}$ \\
\hline
\end{tabular}


Ethnobotany Research and Applications

\begin{tabular}{|c|c|c|c|c|c|c|c|c|}
\hline $\begin{array}{l}\text { Dregea volubilis (L.f.) } \\
\text { Benth. ex Hook.f. } \\
\text { (Apocynaceae) } \\
\end{array}$ & $\begin{array}{l}\text { ekot/ } \\
\text { harandodi }\end{array}$ & brhatpurva & 763 & StemBark & swarasa (juice) & $\begin{array}{l}\text { sarpadansha (Snake } \\
\text { bite) }\end{array}$ & $30-50 \mathrm{ml}$ & $\begin{array}{l}\text { Administer orally immediately } \\
\text { after the snake bite. (Nadkarni } \\
\text { 1999) }\end{array}$ \\
\hline $\begin{array}{l}\text { Embelia tsjeriam-cottam } \\
\text { (Roem. \& Schult.) A. DC. } \\
\text { (Primulaceae) }\end{array}$ & $\begin{array}{l}\text { phatangali/a } \\
\text { mbattingali }\end{array}$ & vidang bhed & 14600 & Root & swarasa (juice) & $\begin{array}{l}\text { sarpadansha (Snake } \\
\text { bite) }\end{array}$ & $2-3 \mathrm{ml}$ & $\begin{array}{l}\text { Instill } 2 \text { drops in the nostrils } \\
\text { twice a day and also apply } \\
\text { juice locally over the forehead. } \\
\text { (Vaidya 1999) }\end{array}$ \\
\hline $\begin{array}{l}\text { Embelia tsjeriam-cottam } \\
\text { (Roem. \& Schult.) A. DC. } \\
\text { (Primulaceae) }\end{array}$ & $\begin{array}{l}\text { phatangali/a } \\
\text { mbattingali }\end{array}$ & vidang bhed & 14600 & Stem Bark & swarasa (juice) & $\begin{array}{l}\text { sarpadansha (Snake } \\
\text { bite), shirashula (head } \\
\text { ache) }\end{array}$ & $1-2$ drop & $\begin{array}{l}\text { Instill juice in the nostrils } \\
\text { immediately after the snake } \\
\text { bite or for headache. } \\
\text { Precaution: do not use more } \\
\text { than two drops as it causes } \\
\text { nasal bleeding. (Vaidya 1999) }\end{array}$ \\
\hline $\begin{array}{l}\text { Ensete superbum (Roxb.) } \\
\text { Cheesman (Musaceae) }\end{array}$ & $\begin{array}{l}\text { rankeli// } \\
\text { kavadar }\end{array}$ & $\begin{array}{l}\text { van kadali/ } \\
\text { bahubija }\end{array}$ & 155 & Root & swarasa (juice) & $\begin{array}{l}\text { mutrashmari } \\
\text { (urolithiasis/ renal } \\
\text { calculi) }\end{array}$ & $30-50 \mathrm{ml}$ & $\begin{array}{l}\text { Administer internally twice a } \\
\text { day for } 8 \text { days. (Sharma et al. } \\
\text { 2002) }\end{array}$ \\
\hline $\begin{array}{l}\text { Ensete superbum (Roxb.) } \\
\text { Cheesman (Musaceae) }\end{array}$ & $\begin{array}{l}\text { rankeli/ } \\
\text { kavadar }\end{array}$ & $\begin{array}{l}\text { van kadali/ } \\
\text { bahubija }\end{array}$ & 155 & Root & churna (powder) & $\begin{array}{l}\text { shwan dansha (dog } \\
\text { bite) }\end{array}$ & $3-5 \mathrm{gm}$ & $\begin{array}{l}\text { Administer powder with water } \\
\text { for 3-6 days. (Sethiya et } \\
\text { al.2019) }\end{array}$ \\
\hline $\begin{array}{l}\text { Euphorbia neriifolia L. } \\
\text { (Euphorbiaceae) }\end{array}$ & sabar & snuhi & 14032 & Aerial part & swarasa (juice) & $\begin{array}{l}\text { karnashula (ear ache), } \\
\text { kasa (Cough), tamak } \\
\text { shwasa (Bronchial } \\
\text { asthma) }\end{array}$ & $5-10 \mathrm{ml}$ & $\begin{array}{l}\text { Collect fresh stem and roast on } \\
\text { burning coal until it becomes } \\
\text { soft, remove its spine, and } \\
\text { extract juice by squeezing it. } \\
\text { Administered orally in tamak } \\
\text { shwas and kasa and instill 1-2 } \\
\text { drops in the ear in case of } \\
\text { karnashula- earache twice a } \\
\text { day for } 4-5 \text { days. (Sharma } \\
2013, \text { Nadkarni. 1999, Desai } \\
\text { 2015) }\end{array}$ \\
\hline $\begin{array}{l}\text { Ficus racemosa } \mathrm{L} . \\
\text { (Moraceae) }\end{array}$ & umbar & udumbar & 14135 & Root & $\begin{array}{l}\text { swarasa } \\
\text { (juice) }\end{array}$ & $\begin{array}{l}\text { anartava/aartav } \\
\text { (Amenorrhea) }\end{array}$ & $30-50 \mathrm{ml}$ & $\begin{array}{l}\text { Administer internally twice a } \\
\text { day for } 2 \text { days before the } \\
\text { expected date of menses on } \\
\text { empty stomach. (Anonymous. } \\
\text { 1999) }\end{array}$ \\
\hline $\begin{array}{l}\text { Ficus religiosa } \mathrm{L} . \\
\text { (Moraceae) }\end{array}$ & pimpal & ashwattha & 2518 & Stem Bark & $\begin{array}{l}\text { swarasa } \\
\text { (juice) }\end{array}$ & $\begin{array}{l}\begin{array}{l}\text { raktamutrata } \\
\text { (haematuria) }\end{array} \\
\end{array}$ & $30-50 \mathrm{ml}$ & $\begin{array}{l}\text { Administer orally twice a day } \\
\text { for } 3 \text { days. (Vaidya 1999) }\end{array}$ \\
\hline $\begin{array}{l}\text { Gardenia turgida Roxb. } \\
\text { (Rubiaceae) }\end{array}$ & pendharun & $\begin{array}{l}\text { bharangi, } \\
\text { mahapindi }\end{array}$ & 14391 & Seed & $\begin{array}{l}\text { dravana } \\
\text { (solution) }\end{array}$ & $\begin{array}{l}\text { balatisara (Diarrhoea } \\
\text { in children) }\end{array}$ & $30 \mathrm{ml}$ & $\begin{array}{l}\text { Mix a cup of water in the seed } \\
\text { powder and filter it after } 5 \text { min. } \\
\text { Administer orally twice a day } \\
\text { for } 3 \text { days. (Uniyal and Tiwari, } \\
\text { 1993) }\end{array}$ \\
\hline $\begin{array}{l}\text { Garuga pinnata Roxb. } \\
\text { (Burseraceae) }\end{array}$ & kakad & golika & 14402 & Stem bark & kalka (paste) & $\begin{array}{l}\text { shotha } \\
\text { (Localised Oedema) } \\
\text { sandhishula (joint }\end{array}$ & As required & $\begin{array}{l}\text { Apply the warm paste over the } \\
\text { affected area; once in a day for } \\
2-3 \text { days (Suneetha et al. 2011) }\end{array}$ \\
\hline
\end{tabular}




\begin{tabular}{|c|c|c|c|c|c|c|c|c|}
\hline & & & & & & $\begin{array}{l}\text { pain) ; sandhishotha } \\
\text { (swelling in the joints) }\end{array}$ & & \\
\hline $\begin{array}{l}\text { Gloriosa superba L. } \\
\text { (Colchicaceae) }\end{array}$ & kalalavi & langali & 14087 & Rhizome & kalka (paste) & $\begin{array}{l}\text { dushtvrana of animal } \\
\text { (Chronic ulcer of } \\
\text { animal ) }\end{array}$ & $\begin{array}{l}1 \text { inch piece of } \\
\text { rhizome }\end{array}$ & $\begin{array}{l}\text { Mix1 inch piece of rhizome in } \\
\text { the fodder of animals once a } \\
\text { day. (Sharma et al. 2001, } \\
\text { Nadkarni 1999) }\end{array}$ \\
\hline $\begin{array}{l}\text { Gmelina arborea L. } \\
\text { (Lamiaceae) }\end{array}$ & shiwan & gambhari & 14204 & Twig & twig & $\begin{array}{l}\text { bhram } \\
\text { (giddiness) }\end{array}$ & As required & $\begin{array}{l}\text { A necklace is prepared from } \\
\text { linch pieces of tender twigs } \\
\text { and kept in the patient's neck } \\
\text { to prevent giddiness as and } \\
\text { when required (Sharma 2013) }\end{array}$ \\
\hline $\begin{array}{l}\text { Gymnema sylvestre } \\
\text { (Retz.) R.Br. ex Sm. } \\
\text { (Apocynaceae) }\end{array}$ & bedaki & meshasringi & 2521 & Leaf & churna (powder) & $\begin{array}{l}\text { madhumeha } \\
\text { (diabetes) }\end{array}$ & $2 \mathrm{gm}$ & $\begin{array}{l}\text { Administer orally once in a } \\
\text { day, empty stomach, for } 10 \\
\text { days. (Nadkarni 1999, } \\
\text { Anonymous 1999) } \\
\end{array}$ \\
\hline $\begin{array}{l}\text { Helicteres isora L. } \\
\text { (Sterculiaceae) }\end{array}$ & murud sheng & avartani & 14281 & Pod & churna (powder) & $\begin{array}{l}\text { udarshula } \\
\text { (abdominal colic) }\end{array}$ & $3-5 \mathrm{gm}$ & $\begin{array}{l}\text { Administer fine powder of } \\
\text { dried pods orally with water } \\
\text { twice a day for } 2-3 \text { day, once } \\
\text { on empty stomach (Sharma } \\
\text { 2013) }\end{array}$ \\
\hline $\begin{array}{l}\text { Hemidesmus indicus (L.) } \\
\text { R. Br. ex Schult. } \\
\text { (Apocynaceae) }\end{array}$ & sariva & sariva & 14346 & Root & root & $\begin{array}{l}\text { udarshula (abdominal } \\
\text { colic) }\end{array}$ & $\begin{array}{l}2-3 \text { inch piece of } \\
\text { root }\end{array}$ & $\begin{array}{l}\text { Masticate 2-3 inch piece of } \\
\text { root, immediately after } \\
\text { abdominal pain. (Desai 2015) }\end{array}$ \\
\hline $\begin{array}{l}\text { Heterophragma } \\
\text { quadriloculare (Roxb.) K.S } \\
\text { chum. (Bignoniaceae) }\end{array}$ & $\begin{array}{l}\text { varas/ } \\
\text { varasa }\end{array}$ & varas & 14380 & Leaf & $\begin{array}{l}\text { swarasa } \\
\text { (juice) }\end{array}$ & alaji (Tinea pedia) & As required & $\begin{array}{l}\text { Apply the juice on sore foot } \\
\text { once in a day. (Sharma 1998, } \\
\text { Surana et al. 2016) }\end{array}$ \\
\hline $\begin{array}{l}\text { Heterophragma } \\
\text { quadriloculare (Roxb.) } \\
\text { K.Schum. (Bignoniaceae) }\end{array}$ & $\begin{array}{l}\text { varas/ } \\
\text { varasa }\end{array}$ & varas & 14380 & Leaf & kalka (paste) & alaji (Tinea pedia) & As required & $\begin{array}{l}\text { Apply the paste locally on the } \\
\text { affected area twice a day for } 2 \text { - } \\
3 \text { days. (Sharma 1998) }\end{array}$ \\
\hline $\begin{array}{l}\text { Holarrhena pubescens } \\
\text { Wall. ex G. Don } \\
\text { (Apocynaceae) }\end{array}$ & kuda & kutaj & 14084 & Sten Bark & swarasa (juice) & $\begin{array}{l}\text { atisara (Diarrhoea) } \\
\text { sarpadansha (Snake } \\
\text { bite) }\end{array}$ & $30-50 \mathrm{ml}$. & $\begin{array}{l}\text { Administer orally twice a day } \\
\text { for 2-3 days; immediately after } \\
\text { the snake bite. (Desai 2015, } \\
\text { Dey \& De 2012) }\end{array}$ \\
\hline $\begin{array}{l}\text { Holarrhena pubescens } \\
\text { Wall. ex G.Don } \\
\text { (Apocynaceae) }\end{array}$ & kuda & kutaj & 14084 & Sten Bark & churna (powder) & $\begin{array}{l}\text { mutrashmari } \\
\text { (urolithiasis/ urinary } \\
\text { calculi), kamala } \\
\text { (Jaundice), } \\
\text { madhumeha } \\
\text { (Diabetes) } \\
\end{array}$ & & $\begin{array}{l}\text { Administer powder internally } \\
\text { with water, twice a day for } 3 \\
\text { days on an empty stomach. } \\
\text { (Vaidya 1999) }\end{array}$ \\
\hline $\begin{array}{l}\text { Holarrhena pubescens } \\
\text { Wall. ex G.Don } \\
\text { (Apocynaceae) }\end{array}$ & kuda & kutaj & 14084 & Root & swarasa (juice) & $\begin{array}{l}\text { udarshula (abdominal } \\
\text { colic) } \\
\text { mutrashmari (Renal } \\
\text { Calculi) } \\
\end{array}$ & $\begin{array}{l}5 \mathrm{ml} \\
30 \mathrm{ml}\end{array}$ & $\begin{array}{l}\text { Administer orally when } \\
\text { requires on empty stomach } \\
\text { early in the morning for 4-5 } \\
\text { days. (Vaidya 1999) }\end{array}$ \\
\hline $\begin{array}{l}\text { Holostemma ada-kodien } \\
\text { Schult. }\end{array}$ & shiricha vel & arkapushpi & 14636 & Fruit & $\begin{array}{l}\text { mashi } \\
\text { (ash) }\end{array}$ & $\begin{array}{l}\text { kasa } \\
\text { (cough) }\end{array}$ & $500 \mathrm{mg}$ & $\begin{array}{l}\text { Collect the fruit and break it to } \\
\text { get the fibre cotton and burn it }\end{array}$ \\
\hline
\end{tabular}




\begin{tabular}{|c|c|c|c|c|c|c|c|c|}
\hline (Apocynaceae) & & & & & & & & $\begin{array}{l}\text { to get ash. In } 500 \mathrm{mg} \text { Ash, add } \\
5 \mathrm{ml} \text { of honey. Administer } \\
\text { orally by licking twice a day for } \\
\text { 3-4 days. (Vaidya 1999) }\end{array}$ \\
\hline $\begin{array}{l}\text { Jatropha curcas L. } \\
\text { (Euphorbiaceae) }\end{array}$ & chadarjyot & dravanti & 14072 & Latex & latex & vrana (Wound) & $5-10 \mathrm{ml}$ & $\begin{array}{l}\text { Collect the latex from the } \\
\text { aerial part and apply over } \\
\text { wounds for 2-3 days for } \\
\text { cleansing. (Nadkarni 1999) }\end{array}$ \\
\hline $\begin{array}{l}\text { Jatropha curcas L. } \\
\text { (Euphorbiaceae) }\end{array}$ & chadarjyot & dravanti & 14072 & $\begin{array}{l}\text { Tender } \\
\text { Root / } \\
\text { Stem Bark }\end{array}$ & $\begin{array}{l}\text { swarasa } \\
\text { (juice) }\end{array}$ & $\begin{array}{l}\text { sarpadansha } \\
\text { (Snake bite) }\end{array}$ & $100 \mathrm{ml}$ & $\begin{array}{l}\text { Administer orally immediately } \\
\text { after a snake bite (Sekhar et al. } \\
\text { 2014) }\end{array}$ \\
\hline $\begin{array}{l}\text { Jatropha curcas L. } \\
\text { (Euphorbiaceae) }\end{array}$ & chadarjyot & dravanti & 14072 & Stem & $\begin{array}{l}\text { dantashula } \\
\text { (tooth ache) }\end{array}$ & As required & & $\begin{array}{l}\text { Warm the stem on fire and } \\
\text { peel the skin off. Use a } \\
\text { toothbrush once a day. } \\
\text { (Anonymous 1999) }\end{array}$ \\
\hline $\begin{array}{l}\text { Lawsonia inermis L. } \\
\text { (Lythraceae) }\end{array}$ & mehandi & madayntika & 14360 & Leaf & swarasa (juice) & kamala (Jaundice) & $30 \mathrm{ml}$ & $\begin{array}{l}\text { Add a glass of cow's milk in } \\
\text { the juice and administer } \\
\text { internally twice a day for two } \\
\text { days. (Sharma et al. 2001, } \\
\text { Vaidya 1999) }\end{array}$ \\
\hline $\begin{array}{l}\text { Leea macrophylla Roxb. } \\
\text { ex Hornem. (Vitaceae) }\end{array}$ & dinda & hastikarna & 14614 & Tuber & kalka (paste) & $\begin{array}{l}\text { gatha/ granthi } \\
\text { (Tumor) } \\
\text { pidika (boils/ } \\
\text { Furuncles) } \\
\end{array}$ & As required & $\begin{array}{l}\text { Apply locally on the affected } \\
\text { area, once a day for } 8 \text { days. } \\
\text { (Desai 2015, Nadkarni 1999) }\end{array}$ \\
\hline $\begin{array}{l}\text { Madhuca indica J.F.Gmel. } \\
\text { (Sapotaceae) }\end{array}$ & moha & madhuka & 14126 & Seed & churna (powder) & $\begin{array}{l}\text { shirashula } \\
\text { (headache) }\end{array}$ & As required & $\begin{array}{l}\text { Inhale powder through nostrils } \\
\text { once a day. (Sharma 2013) }\end{array}$ \\
\hline $\begin{array}{l}\text { Mangifera indica } \mathrm{L} . \\
\text { (Anacardiaceae) }\end{array}$ & amba & amra & 2653 & Stem Bark & swarasa (juice) & $\begin{array}{l}\text { arsha } \\
\text { (Piles/ haemorrhoids) }\end{array}$ & $30 \mathrm{ml}$ & $\begin{array}{l}\text { Administer orally once in a day } \\
\text { on empty stomach for } 2 \text { days. } \\
\text { (Desai 2015) }\end{array}$ \\
\hline $\begin{array}{l}\text { Momordica dioica Roxb. } \\
\text { ex Willd. (Cucurbitaceae) }\end{array}$ & kartuli & karkotaki & 14012 & Root & kalka (paste) & $\begin{array}{l}\text { madhumehavrana } \\
\text { (Diabeties wound) }\end{array}$ & $1-2$ inch piece & $\begin{array}{l}\text { Apply on the affected area } \\
\text { once a day, for } 15 \text { days. } \\
\text { (Vaidya 1999) }\end{array}$ \\
\hline $\begin{array}{l}\text { Moringa oleifera Lam. } \\
\text { (Moringaceae) }\end{array}$ & shevaga & shigru & 2019 & Stem Bark & stem bark & $\begin{array}{l}\text { udarshula (Abdominal } \\
\text { colic) }\end{array}$ & As required & $\begin{array}{l}\text { Keep fresh stem bark on the } \\
\text { stomach while lying down or } \\
\text { tie with a cloth while moving } \\
\text { or working for } 30 \text { min. } \\
\text { (Nadkarni 1999) }\end{array}$ \\
\hline $\begin{array}{l}\text { Moringa oleifera Lam. } \\
\text { (Moringaceae) }\end{array}$ & shevaga & shigru & 2019 & Leaf & $\begin{array}{l}\text { swarasa } \\
\text { (juice) }\end{array}$ & $\begin{array}{l}\text { abhishyanda } \\
\text { (Conjunctivitis) }\end{array}$ & 2 drops & $\begin{array}{l}\text { Instil juice in infected eyes } \\
\text { twice a day for two days. } \\
\text { (Vaidya 1999, Nadkarni 1999) }\end{array}$ \\
\hline $\begin{array}{l}\text { Myristica fragrans Houtt. } \\
\text { (Myristicaceae) }\end{array}$ & jayphal & jatiphal & 3783 & $\begin{array}{l}\text { Fruit, } \\
\text { Flower }\end{array}$ & churna (powder) & $\begin{array}{l}\text { vandyatva (female } \\
\text { Infertility) }\end{array}$ & $5 \mathrm{gm}$ & $\begin{array}{l}\text { Administer orally on empty } \\
\text { stomach for } 5 \text { days. (Nadkarni } \\
\text { 1999) }\end{array}$ \\
\hline $\begin{array}{l}\text { Ocimum tenuiflorum } \mathrm{L} . \\
\text { (Lamiaceae) }\end{array}$ & tulashi & krishnatulasi & 2680 & Leaf & leaves & $\begin{array}{l}\text { vruschik dansha } \\
\text { (scorpion sting) }\end{array}$ & 5-6 leaves & $\begin{array}{l}\text { Take a handful of tulasi leaves } \\
\text { and rub them on hands and } \\
\text { chant the mantra. Tell the }\end{array}$ \\
\hline
\end{tabular}




\begin{tabular}{|c|c|c|c|c|c|c|c|c|}
\hline & & & & & & & & $\begin{array}{l}\text { patient to look at the leaves. } \\
\text { (Belief) (Vedavathy et al.1997) }\end{array}$ \\
\hline $\begin{array}{l}\text { Operculina turpethum (L.) } \\
\text { Silva Manso } \\
\text { (Convolvulaceae) }\end{array}$ & tinshira & trivrit & 4223 & Stem & tender fresh stem & $\begin{array}{l}\text { kasa (Cough)/ } \\
\text { yakshma } \\
\text { (tuberculosis/ } \\
\text { Consumption) }\end{array}$ & $3-4$ inch & $\begin{array}{l}\text { Masticate thrice a day for 2-3 } \\
\text { days. (Vaidya 1999) }\end{array}$ \\
\hline Oxide of Iron $\left(\mathrm{Fe}_{2} \mathrm{O}_{3}\right)$. & geru & gairik & MS-457 & Mineral & kalka (paste) & $\begin{array}{l}\text { visarpa } \\
\text { (herpes zoster) }\end{array}$ & As required & $\begin{array}{l}\text { Prepare a paste by mixing curd } \\
\text { (1/4 kg) add } 3 \text { teaspoons of } \\
\text { geru. Apply the paste on the } \\
\text { affected area. (by chanting the } \\
\text { mantra). (Bhavmishra 2006) }\end{array}$ \\
\hline $\begin{array}{l}\text { Peucedanum grande } \\
\text { C.B.Clarke (Apiaceae) }\end{array}$ & baphali & baspika & 14220 & Seed & churna (powder) & $\begin{array}{l}\text { adhmana } \\
\text { (flatulence) }\end{array}$ & $2-3 \mathrm{gm}$ & $\begin{array}{l}\text { Administer orally, once a day } \\
\text { for } 3 \text { days, on empty stomach } \\
\text { in the morning. ( Vaidya 1999) }\end{array}$ \\
\hline $\begin{array}{l}\text { Phoenix sylvestris (L.) } \\
\text { Roxb. (Arecaceae) }\end{array}$ & shindoli & khajur bheda & 859 & Resin & vati (pills) & $\begin{array}{l}\text { mutrashmari } \\
\text { (Urolithiasis) }\end{array}$ & $5 \mathrm{tab} / 500 \mathrm{mg}$ & $\begin{array}{l}\text { Administer internally early in } \\
\text { the morning on empty } \\
\text { stomach for } 5 \text { days. (Nadkarni } \\
\text { 1999) }\end{array}$ \\
\hline $\begin{array}{l}\text { Plumbago zeylanica } \mathrm{L} \text {. } \\
\text { (Plumbaginaceae). }\end{array}$ & chitrak & chitrak & 14275 & Root & churna (powder) & $\begin{array}{l}\text { vaivarnya } \\
\text { (Discoloration of skin)- } \\
\text { twakvikar (Skin } \\
\text { disease) }\end{array}$ & As required & $\begin{array}{l}\text { Apply externally on the } \\
\text { affected area for } 10 \text { minutes, } \\
\text { once in a day, repeat if } \\
\text { required. (Nadkarni 1999, } \\
\text { Anonymous 1999) }\end{array}$ \\
\hline $\begin{array}{l}\text { Plumeria obtusa L. } \\
\text { (Apocynaceae) }\end{array}$ & chapha & $\begin{array}{l}\text { champaka } \\
\text { bhed }\end{array}$ & 3958 & Pod & kalka (paste) & $\begin{array}{l}\text { sarpadansha } \\
\text { (snake bite) }\end{array}$ & One teaspoon (Stat) & $\begin{array}{l}\text { Mix one teaspoonful of honey } \\
\text { in the paste and administer it } \\
\text { orally to the patient once a } \\
\text { day. (Nadkarni 1999) }\end{array}$ \\
\hline $\begin{array}{l}\text { Pogostemon } \\
\text { benghalensis (Burm.f.) } \\
\text { Kuntze (Lamiaceae) }\end{array}$ & fangali & - & 14258 & Leaf & swarasa (juice) & $\begin{array}{l}\text { udarshula } \\
\text { (abdominal colic) }\end{array}$ & $5 \mathrm{ml}$ & $\begin{array}{l}\text { Administer orally twice a day } \\
\text { on empty stomach. (Sharma } \\
1998 \text { ) }\end{array}$ \\
\hline $\begin{array}{l}\text { Pogostemon } \\
\text { benghalensis (Burm.f.) } \\
\text { Kuntze (Lamiaceae) }\end{array}$ & fangali & - & 14258 & Root /Leaf & $\begin{array}{l}\text { kalka } \\
\text { (paste) }\end{array}$ & $\begin{array}{l}\text { vrushchik dansha } \\
\text { (Scorpion sting) }\end{array}$ & As required & $\begin{array}{l}\text { Apply locally on the affected } \\
\text { area once in a day. (Nadkarni } \\
\text { 1999, Desai 2015, } \\
\text { Annonymous CSIR 1986) }\end{array}$ \\
\hline $\begin{array}{l}\text { Polyalthia longifolia } \\
\text { (Sonn.) Thwaites } \\
\text { (Annonaceae) }\end{array}$ & $\begin{array}{l}\text { mumbai } \\
\text { ashok }\end{array}$ & kasthadaru & 1803 & Stem Bark & swarasa (juice) & $\begin{array}{l}\text { sweta pradar } \\
\text { (leucorrhoea) }\end{array}$ & $30 \mathrm{ml}$ & $\begin{array}{l}\text { Administer orally once in a } \\
\text { day, for } 2 \text { days. (Vaidya 1999; } \\
\text { Desai } 2015 \text { ) }\end{array}$ \\
\hline $\begin{array}{l}\text { Pongamia pinnata (L.) } \\
\text { Pierre. (Leguminosae) }\end{array}$ & karanja & karanja & 14174 & Stem bark & $\begin{array}{l}\text { kwatha } \\
\text { (decoction) }\end{array}$ & $\begin{array}{l}\text { kamala } \\
\text { (Jaundice) }\end{array}$ & $50-60 \mathrm{ml}$ & $\begin{array}{l}\text { Administer orally twice a day } \\
\text { on empty stomach for 5-6 } \\
\text { days. (Vaidya 1999) }\end{array}$ \\
\hline $\begin{array}{l}\text { Pterocarpus marsupium } \\
\text { Roxb. (Leguminosae) }\end{array}$ & bivala & vijaysara & 14292 & Stem Bark & $\begin{array}{l}\text { phant (hot } \\
\text { infusion) }\end{array}$ & $\begin{array}{l}\text { raktatisara (Bleeding } \\
\text { diarrhoea) }\end{array}$ & $30 \mathrm{ml}$ & $\begin{array}{l}\text { Boil stem bark in water, filter } \\
\text { and administer orally once a } \\
\text { day. (Desai 2015) }\end{array}$ \\
\hline $\begin{array}{l}\text { Pterocarpus marsupium } \\
\text { Roxb. (Leguminosae) }\end{array}$ & bivala & vijaysara & 14292 & Stem Bark & churna (powder) & $\begin{array}{l}\text { haridrameha/ } \\
\text { pitamutrata } \\
\text { raktamutrata / }\end{array}$ & $5 \mathrm{gm}$ & $\begin{array}{l}\text { Prepare fine powder of dried } \\
\text { stem bark by pounding. } \\
\text { Administer fine powder with }\end{array}$ \\
\hline
\end{tabular}




\begin{tabular}{|c|c|c|c|c|c|c|c|c|}
\hline & & & & & & $\begin{array}{l}\text { raktajmeha (yellowish } \\
\text { urination/ Haematuria) }\end{array}$ & & $\begin{array}{l}\text { water, twice a day on an empty } \\
\text { stomach for } 1 \text { day. (Sharma } \\
\text { 2013) }\end{array}$ \\
\hline $\begin{array}{l}\text { Radermachera xylocarpa } \\
\text { (Roxb.) Roxb. ex K. } \\
\text { Schum. (Bignoniaceae) }\end{array}$ & khara shinga & shwetapatala & 14228 & Stem Bark & churna (powder) & $\begin{array}{l}\text { dadru (Ringworm } \\
\text { infection)-tvakvikar } \\
\text { (Skin disease) }\end{array}$ & As require & $\begin{array}{l}\text { Prepare the powder of dried } \\
(150-200 \mathrm{~g}) \text { stem bark and boil } \\
\text { it in } 1-2 \text { lit water, add this in } \\
\text { warm water and take bath for } \\
2-3 \text { days. (Desai } 2015)\end{array}$ \\
\hline $\begin{array}{l}\text { Ricinus communis L. } \\
\text { (Euphorbiaceae) }\end{array}$ & lal eranda & rakta eranada & 2672 & Leaf & $\begin{array}{l}\text { swarasa } \\
\text { (juice) }\end{array}$ & $\begin{array}{l}\text { ashmari } \\
\text { (urolithiasis/urinary } \\
\text { calculi) }\end{array}$ & $150 \mathrm{ml}$. & $\begin{array}{l}\text { Extract the juice by crushing 8- } \\
10 \text { fresh leaves and add } \\
\text { approximately } 1 \text { lit water. } \\
\text { Administer orally thrice a day } \\
\text { for 7-8 days. (Anonymous. } \\
1999 \text { ) }\end{array}$ \\
\hline $\begin{array}{l}\text { Ricinus communis } \mathrm{L} \text {. } \\
\text { (Euphorbiaceae) }\end{array}$ & lal eranda & rakta eranada & 2672 & Root & swarasa (juice) & $\begin{array}{l}\text { mutrakrucchra } \\
\text { (Painful micturation/ } \\
\text { dysuria)/ raktamutrata } \\
\text { (Haematouria) }\end{array}$ & $30 \mathrm{ml}$ & $\begin{array}{l}\text { Administer orally once a day } \\
\text { on an empty stomach for } 3 \\
\text { days. } \\
\text { (Sharma 2013) }\end{array}$ \\
\hline $\begin{array}{l}\text { Sapindus emarginatus } \\
\text { Vahl } \\
\text { (Sapindaceae) }\end{array}$ & ritha & arishtaka & 14252 & Root & $\begin{array}{l}\text { dravana } \\
\text { (solution) }\end{array}$ & $\begin{array}{l}\text { udarshula (Abdominal } \\
\text { colic) }\end{array}$ & $10-20 \mathrm{ml}$ & $\begin{array}{l}\text { Add 2-3 spoonful of water in } \\
\text { the powder and administer it } \\
\text { internally for 2-3 days. } \\
\text { (Nadkarni 1999) }\end{array}$ \\
\hline $\begin{array}{l}\text { Sapindus emarginatus } \\
\text { Vahl } \\
\text { (Sapindaceae) }\end{array}$ & ritha & arishtaka & 14252 & Fruit & $\begin{array}{l}\text { dravana } \\
\text { (solution) }\end{array}$ & $\begin{array}{l}\text { malavibandha } \\
\text { (Constipation) }\end{array}$ & $\begin{array}{l}10-15 \mathrm{ml} \\
\text { (paediatric)/ } 30 \mathrm{ml} \\
\text { (Adult) }\end{array}$ & $\begin{array}{l}\text { Prepare the solution by } \\
\text { crushing the rind of single fruit } \\
\text { in approx. } 100 \mathrm{ml} \text { ( } 1 \text { glass) } \\
\text { water and filter. Administer the } \\
\text { squeezed filtrate orally at } \\
\text { bedtime. (Nadkarni 1999) }\end{array}$ \\
\hline $\begin{array}{l}\text { Sesbania sesban (L.) Merr. } \\
\text { (Leguminosae) }\end{array}$ & dedhara & itakata & 3748 & Latex & latex & $\begin{array}{l}\text { kumbhika/anjananam } \\
\text { ika } \\
\text { (stye) }\end{array}$ & As required & $\begin{array}{l}\text { Collect the yellowish watery } \\
\text { latex obtained from the base } \\
\text { of the leaf and apply locally on } \\
\text { the style. Once a day for } 2-3 \\
\text { days. (Anonymous. 1999) }\end{array}$ \\
\hline $\begin{array}{l}\text { Sida rhombifolia L. } \\
\text { Malvaceae }\end{array}$ & chikana & bala bhed & 780 & Leaf & $\begin{array}{l}\text { swarasa } \\
\text { (juice) }\end{array}$ & $\begin{array}{l}\text { pitika (pimples) / } \\
\text { shotha swelling }\end{array}$ & As required & $\begin{array}{l}\text { Apply on face, once a day for } \\
\text { 5-7 days. (Dwivedi 2008, } \\
\text { Sharma 2013) }\end{array}$ \\
\hline $\begin{array}{l}\text { Solanum virginianum } \mathrm{L} \text {. } \\
\text { (Solanaceae) }\end{array}$ & ringani & kantakari & 2371 & $\begin{array}{l}\text { Riped } \\
\text { Fruit }\end{array}$ & $\begin{array}{l}\text { yavkut (coarse } \\
\text { podwer) }\end{array}$ & $\begin{array}{l}\text { dantakrumi (Dental } \\
\text { caries) }\end{array}$ & As required & $\begin{array}{l}\text { Crush the dried ripe fruit and } \\
\text { heat it over the iron pan and } \\
\text { inhale the fumes through the } \\
\text { mouth to expose dental caries. } \\
\text { Once a day for } 3-5 \text { days } \\
\text { (Sharma et al. } 2002 \text {, Nadkarni } \\
\text { 1999, Anonymous 1999) }\end{array}$ \\
\hline $\begin{array}{l}\text { Solanum virginianum } \mathrm{L} \text {. } \\
\text { (Solanaceae) }\end{array}$ & ringani & kantakari & 2371 & Fruit & mashi (ash) & $\begin{array}{l}\text { arsha (Piles/ } \\
\text { haemorrihoids) }\end{array}$ & As required & $\begin{array}{l}\text { Prepare ash of dried fruits by } \\
\text { burning and add a sufficient } \\
\text { quantity of coconut oil to get }\end{array}$ \\
\hline
\end{tabular}




\begin{tabular}{|c|c|c|c|c|c|c|c|c|}
\hline & & & & & & & & $\begin{array}{l}\text { the paste, apply locally on the } \\
\text { affected area once a day for } 8 \text { - } \\
10 \text { days. (Sharma et al.2002, } \\
\text { Nadkarni 1999;) }\end{array}$ \\
\hline $\begin{array}{l}\text { Solena amplexicaulis } \\
\text { (Lam.) Gandhi. } \\
\text { (Cucurbitaceae) }\end{array}$ & gometi & sukakandah & 14022 & Root & root & $\begin{array}{l}\text { kshudhakshyaya (loss } \\
\text { of appetite) } \\
\text { agnimandhya } \\
\text { (loss of appetite) }\end{array}$ & 1-2 inch piece & $\begin{array}{l}\text { Roast the root on burning } \\
\text { coals and consume for a day. } \\
\text { (Natarajan \& Paulsen 2000) }\end{array}$ \\
\hline $\begin{array}{l}\text { Solena amplexicaulis } \\
\text { (Lam.) Gandhi. } \\
\text { (Cucurbitaceae) }\end{array}$ & gometi & sukakandah & 14022 & Fruit & $\begin{array}{l}\text { swarasa } \\
\text { (juice)/ } \\
\text { dravana } \\
\text { (solution) }\end{array}$ & $\begin{array}{l}\text { karnashula } \\
\text { (ear ache/Otalgia) }\end{array}$ & 2 drops in a ear & $\begin{array}{l}\text { Juice extracted from the fresh } \\
\text { fruit or soak dried fruit powder } \\
\text { in water and that solution is to } \\
\text { be instilled in the ear as and } \\
\text { when required. (Patil 2006) }\end{array}$ \\
\hline $\begin{array}{l}\text { Sphaeranthus indicus L. } \\
\text { (Compositae) }\end{array}$ & mundi & mundi & 14363 & Flower & $\begin{array}{l}\text { hima } \\
\text { (cold infusion)/ } \\
\text { fresh flower }\end{array}$ & sthoulya (obesity) & $150 \mathrm{ml}$ & $\begin{array}{l}\text { Soak 7-8 dried flowers in water } \\
\text { overnight and crush on the } \\
\text { next morning; filter it and } \\
\text { administered the filtrate orally/ } \\
\text { consume } 7-8 \text { fresh flowers } \\
\text { once a day for } 30 \text { days on } \\
\text { empty stomach. (Bhavmishra } \\
\text { 2006) }\end{array}$ \\
\hline $\begin{array}{l}\text { Syzygium cumini (L.) } \\
\text { Skeels. (Myrtaceae) }\end{array}$ & jambhul & jambu & 669 & Seed & churna (powder) & $\begin{array}{l}\text { madhumeha } \\
\text { (Diabetes) } \\
\text { Antraopucchashotha } \\
\text { (Appendicitis) } \\
\end{array}$ & $5 \mathrm{gm}$ & $\begin{array}{l}\text { Administer internally with } \\
\text { water twice a day for } 7 \text { days. } \\
\text { (Desai 2015, Anonymous. } \\
\text { 1999, Vaidya 1999) }\end{array}$ \\
\hline $\begin{array}{l}\text { Tectona grandis L.f. } \\
\text { (Lamiaceae) }\end{array}$ & sag & shaka & 14048 & Stem Bark & swarasa (juice) & $\begin{array}{l}\text { udarshula (abdominal } \\
\text { colic) }\end{array}$ & 3-4 gm & $\begin{array}{l}\text { Extract the juice of stem bark } \\
\text { by crushing and adding a } \\
\text { sufficient quantity of water. } \\
\text { Administer orally once a day as } \\
\text { and when required. (Nadkarni } \\
\text { 1999, Desai 2015) }\end{array}$ \\
\hline $\begin{array}{l}\text { Tectona grandis L.f. } \\
\text { (Lamiaceae) }\end{array}$ & saga & shaka & 14048 & Seed & $\begin{array}{l}\text { churna } \\
\text { (powder) }\end{array}$ & $\begin{array}{l}\text { ashmari } \\
\text { (Urolithiasis/ urinary } \\
\text { calculi) }\end{array}$ & $3 \mathrm{gm}$ & $\begin{array}{l}\text { Administer orally with } \\
\text { lukewarm water, twice a day } \\
\text { for } 2-3 \text { days on empty } \\
\text { stomach. (Vaidya 1999) }\end{array}$ \\
\hline $\begin{array}{l}\text { Tectona grandis L.f. } \\
\text { (Lamiaceae) }\end{array}$ & saga & shaka & 14048 & Seed & $\begin{array}{l}\text { dravana } \\
\text { (solution) }\end{array}$ & $\begin{array}{l}\text { mutrashmari } \\
\text { (urolithiasis/ renal } \\
\text { calculi) }\end{array}$ & $50 \mathrm{ml}$ & $\begin{array}{l}\text { Crush } 4-5 \text { seeds and soak in } 50 \\
\text { ml water and filter it. } \\
\text { Administer internally thrice a } \\
\text { day before food for 3-4 days. } \\
\text { (Vaidya 1999) }\end{array}$ \\
\hline $\begin{array}{l}\text { Terminalia bellirica } \\
\text { (Gaertn.) Roxb. } \\
\text { (Combretaceae) } \\
\end{array}$ & behada & bibhitaki & 4144 & Fruit & fruit & $\begin{array}{l}\text { udarshula (abdominal } \\
\text { colic) }\end{array}$ & 1 fruit & $\begin{array}{l}\text { Remove the rind of the fruit } \\
\text { and consume once a day for 2- } \\
3 \text { days. (Sharma et al. 2001) }\end{array}$ \\
\hline $\begin{array}{l}\text { Terminalia bellirica } \\
\text { (Gaertn.) Roxb. } \\
\text { (Combretaceae) }\end{array}$ & behada & bibhitaki & 4144 & Fruit & fruit pericarp & anidra (Insomnia) & $3-5 \mathrm{gm}$ & $\begin{array}{l}\text { Collect and masticate the } \\
\text { pericarp of matured fruit }\end{array}$ \\
\hline
\end{tabular}




\begin{tabular}{|c|c|c|c|c|c|c|c|c|}
\hline & & & & & & & & $\begin{array}{l}\text { administer orally once a day, at } \\
\text { bedtime. (Sharma 2013) }\end{array}$ \\
\hline $\begin{array}{l}\text { Terminalia chebula Retz. } \\
\text { (Combretaceae) }\end{array}$ & hirada & haritaki & 2356 & Stem Bark & churna (powder) & kasa (Cough) & $3 \mathrm{gm}$ & $\begin{array}{l}\text { Prepare the fine powder by } \\
\text { pounding dried stem bark. } \\
\text { Administer orally with water, } \\
\text { twice a day for } 3-4 \text { days. } \\
\text { (Bhavmishra 2006) }\end{array}$ \\
\hline $\begin{array}{l}\text { Terminalia tomentosa } \\
\text { Wight \& Arn. } \\
\text { (Combretaceae) }\end{array}$ & $\begin{array}{l}\text { yen/ } \\
\text { ain } \\
\text { arjun sadada }\end{array}$ & arjuna bheda & 3957 & Stem Bark & stem bark & $\begin{array}{l}\text { vrana } \\
\text { (Wound/ulcer) }\end{array}$ & As require & $\begin{array}{l}\text { Keep a piece of fresh stem } \\
\text { bark over the wound for 3-4 } \\
\text { days by bandaging it with a } \\
\text { cloth. (Sharma 2013) }\end{array}$ \\
\hline $\begin{array}{l}\text { Terminalia tomentosa } \\
\text { Wight \& Arn. } \\
\text { (Combretaceae) }\end{array}$ & arjun sadada & arjun & 4071 & Stem Bark & swarasa (juice) & $\begin{array}{l}\text { panduroga (Anemia), } \\
\text { anartava } \\
\text { (Amenorrhoea), } \\
\text { rajodosha (Menstrual } \\
\text { disorders) } \\
\end{array}$ & $5 \mathrm{gm}$ & $\begin{array}{l}\text { Extract the juice of fresh stem } \\
\text { bark. Administer orally on } \\
\text { empty stomach for 20-30 days. } \\
\text { (Sharma 2013) }\end{array}$ \\
\hline $\begin{array}{l}\text { Terminalia tomentosa } \\
\text { Wight \& Arn. } \\
\text { (Combretaceae) }\end{array}$ & $\begin{array}{l}\text { yen/ } \\
\text { ain } \\
\text { arjun sadada }\end{array}$ & arjuna bheda & 3957 & Stem Bark & kalka (paste) & $\begin{array}{l}\text { vrana } \\
\text { (wound/ulcer), } \\
\text { dushtavrana } \\
\text { (chronic ulcer) }\end{array}$ & As required & $\begin{array}{l}\text { Prepare the paste of freshly } \\
\text { collected stem bark. Apply } \\
\text { locally (paste or fine powder) } \\
\text { over the wound. Once in a day, } \\
\text { for } 7-8 \text { days or as required. } \\
\text { (Sharma 2013) }\end{array}$ \\
\hline $\begin{array}{l}\text { Thespesia lampas (Cav.) } \\
\text { Dalzell (Malvaceae) }\end{array}$ & ranbhendi & vanakarpasah & 14099 & Root & $\begin{array}{l}\text { swarasa } \\
\text { (juice) }\end{array}$ & $\begin{array}{l}\text { udarshula } \\
\text { (Abdominal colic) }\end{array}$ & $5 \mathrm{ml}$ & $\begin{array}{l}\text { Administer the filtrate orally as } \\
\text { required. (Pawar \& Patil 2008) }\end{array}$ \\
\hline $\begin{array}{l}\text { Tinospora cordifolia } \\
\text { (Willd.) Miers } \\
\text { (Menispermiaceae) }\end{array}$ & gulvel & guduchi & 14209 & Stem & swarasa (juice) & $\begin{array}{l}\text { jwara (fever), kamala } \\
\text { (Jaundice) }\end{array}$ & $10 \mathrm{ml}$ & $\begin{array}{l}\text { Administer orally twice a day } \\
\text { for 2-3 days. } \\
\text { ( Vaidya 1999, Anonymous } \\
\text { 1999, Sharma et al. 2001) }\end{array}$ \\
\hline $\begin{array}{l}\text { Tinospora cordifolia } \\
\text { (Willd.) Miers } \\
\text { (Menispermiaceae) }\end{array}$ & gulvel & guduchi & 14209 & Stem & $\begin{array}{l}\text { dravana } \\
\text { (solution) }\end{array}$ & $\begin{array}{l}\text { raktasrsha (bleeding } \\
\text { piles) }\end{array}$ & $30 \mathrm{ml}$ & $\begin{array}{l}\text { Soak } 1 \text { tablespoonful powder } \\
\text { (5gm) in a cup of water and } \\
\text { keep for } 10-15 \text { min. Filter and } \\
\text { administer the filtrate on } \\
\text { empty stomach for } 2-3 \text { days. ( } \\
\text { Anonymous. 1999) }\end{array}$ \\
\hline $\begin{array}{l}\text { Tinospora cordifolia } \\
\text { (Willd.) Miers } \\
\text { (Menispermiaceae) }\end{array}$ & gulvel & guduchi & 14209 & Stem & kalka (paste) & $\begin{array}{l}\text { sandhigatvata } \\
\text { (Osteo-arthritis) }\end{array}$ & As required & $\begin{array}{l}\text { Make a fine paste of fresh stem } \\
\text { using the required quantity of } \\
\text { water and apply externally on } \\
\text { affected part once in a day } \\
\text { (Agnivesh 2007) }\end{array}$ \\
\hline $\begin{array}{l}\text { Tridax procumbens (L.) L. } \\
\text { (Compositae) }\end{array}$ & $\begin{array}{l}\text { burada } \\
\text { /jakhamjodi }\end{array}$ & jayantiveda & 3943 & Leaf & swarasa (juice) & $\begin{array}{l}\text { dantshula (Toothache) } \\
\text { vrana } \\
\text { (Wound/ulcer) }\end{array}$ & 2 drops & $\begin{array}{l}\text { Extract the juice of fresh leaves } \\
\text { by crushing. Instil } 2 \text { drops in } \\
\text { the ear of the affected teeth } \\
\text { side. } \\
\text { Apply locally over the wound } \\
\text { once in a day for } 2-3 \text { days. } \\
\text { (Rao \& Henry 1996) }\end{array}$ \\
\hline
\end{tabular}


Ethnobotany Research and Applications

\begin{tabular}{|c|c|c|c|c|c|c|c|c|}
\hline $\begin{array}{l}\text { Vitex negundo L. } \\
\text { (Lamiaceae) }\end{array}$ & nirgundi & nirgundi & 14045 & Leaf & kalka (paste) & $\begin{array}{l}\text { vruschik dansha } \\
\text { (Scorpion sting) } \\
\text { shirashula (Headache) } \\
\text { Dantashula } \\
\text { (Toothache) }\end{array}$ & As required & $\begin{array}{l}\text { Prepare the paste of freshly } \\
\text { collected leaf by crushing on } \\
\text { hands and apply immediately } \\
\text { to the affected area. ( Desai } \\
2015 \text {, Sharma et al. 2001, } \\
\text { Anonymous1999, Vaidya 1999) } \\
\text { Instil 1-2 drops in the nose as } \\
\text { errhine (Nasya) }\end{array}$ \\
\hline $\begin{array}{l}\text { Vitex negundo L. } \\
\text { (Lamiaceae) }\end{array}$ & nirgundi & nirgundi & 14045 & Root & $\begin{array}{l}\text { kwatha } \\
\text { (decoction) }\end{array}$ & $\begin{array}{l}\text { Sandhivat } \\
\text { (Osteoarthritis) }\end{array}$ & $30 \mathrm{ml}$ & $\begin{array}{l}\text { Administer orally once a day } \\
\text { on empty stomach. (Sharma } \\
\text { 2004, Sharma et al. 2001) }\end{array}$ \\
\hline $\begin{array}{l}\text { Dregea volubilis (L.f.) } \\
\text { Benth. ex Hook.f. } \\
\text { (Apocynaceae) }\end{array}$ & karodi & swarna jivanti & 763 & Leaf & $\begin{array}{l}\text { kalka } \\
\text { (paste) }\end{array}$ & $\begin{array}{l}\text { sarpadansha shotha } \\
\text { (Swelling due to snake } \\
\text { bite) }\end{array}$ & As required & $\begin{array}{l}\text { Prepare the paste of freshly } \\
\text { collected leaves by crushing } \\
\text { and rubbing it on hands. Apply } \\
\text { externally on the swelling } \\
\text { developed due to snake bite, } \\
\text { once a day. (Nadakarni 1999) }\end{array}$ \\
\hline $\begin{array}{l}\text { Woodfordia fruticosa (L.) } \\
\text { Kurz. (Lytheraceae) }\end{array}$ & dhayti & dhataki & 14389 & Leaf & leave & jwara (Fever) & $50-100 \mathrm{gm}$ & $\begin{array}{l}\text { Take leaves and boiled in } \\
\text { water and use to take a bath. ( } \\
\text { Sharma 2013) }\end{array}$ \\
\hline $\begin{array}{l}\text { Ziziphus xylopyrus (Retz.) } \\
\text { Willd. (Rhamnaceae) }\end{array}$ & bora & badar & 14222 & Leaf & kalka (paste) & $\begin{array}{l}\text { vruschik dansha } \\
\text { (Scorpion sting) }\end{array}$ & & $\begin{array}{l}\text { Crush the fresh leaf, and apply } \\
\text { over the affected area by } \\
\text { chanting the mantra. (Belief ) } \\
\text { (Nadkarni 1999) }\end{array}$ \\
\hline
\end{tabular}


Table. 3. Medico-ethnobotanical claims of compound formulation reported during the survey of Jawhar and Shahapur Forest division

\begin{tabular}{|c|c|c|c|c|c|c|c|c|}
\hline local name & $\begin{array}{l}\text { Sanskrit } \\
\text { name }\end{array}$ & Botanical Name & $\begin{array}{l}\text { Voucher } \\
\text { specimen } \\
\text { number }\end{array}$ & $\begin{array}{l}\text { Part } \\
\text { Used }\end{array}$ & $\begin{array}{l}\text { Form of } \\
\text { formulation }\end{array}$ & Indication & Dose & Mode of application and validation \\
\hline sabar & snuhi & $\begin{array}{l}\text { Euphorbia nerïfolia L } \\
\text { (Euphorbiaceae) }\end{array}$ & 14032 & $\begin{array}{l}\text { Aerial } \\
\text { Part }\end{array}$ & kalka (Paste) & $\begin{array}{l}\text { shotha } \\
\text { (Swelling) }\end{array}$ & As required & $\begin{array}{l}\text { Take fresh stem of sabar, remove the thorns, } \\
\text { cut vertically into } 2 \text { parts. Apply turmeric } \\
\text { powder on to it and worm. Tie on swelling } \\
\text { and keep it overnight. (Sharma 2013) }\end{array}$ \\
\hline halad & haridra & $\begin{array}{l}\text { Curcuma longa L. } \\
\text { (Zingiberaceae) }\end{array}$ & 2816 & Rhizome & & & & \\
\hline gulvela & guduchi & $\begin{array}{l}\text { Tinospora cordifolia } \\
\text { (Willd.) Miers } \\
\text { (Menispermiaceae) }\end{array}$ & 14207 & Stem & $\begin{array}{l}\text { kwatha } \\
\text { (Decoction }\end{array}$ & $\begin{array}{l}\text { rajyakshma } \\
\text { (Tuberculosis/ } \\
\text { consumption }\end{array}$ & $\begin{array}{l}1 \text { gm Kutaj } \\
\text { powder, } \\
\text { goat urine } \\
10-20 \mathrm{ml} \\
\text { and } 30 \mathrm{ml} \\
\text { Decoction } \\
\text { of Guduchi } \\
\text { stem }\end{array}$ & $\begin{array}{l}\text { Prepare the powder of dry stem bark of } \\
\text { kutaja; Add } 10-20 \mathrm{ml} \text { of goat urine in } 1 \mathrm{gm} \\
\text { kutaj powder mix this solution in } 30 \mathrm{ml} \\
\text { decoction of guduchi (prepared by boiling } \\
3-5 \text { gm of powder in approx. } 100 \mathrm{ml} \text { water } \\
\text { and reduce it to the half quantity). } \\
\text { Administer the mixture once a day for } 15 \\
\text { days, on empty stomach (Agnivesh 2007) }\end{array}$ \\
\hline safed kuda & kutaj & $\begin{array}{l}\text { Holarrhena } \\
\text { pubescens Wall. ex G. } \\
\text { Don (Apocynaceae) }\end{array}$ & 14082 & $\begin{array}{l}\text { Stem } \\
\text { Bark }\end{array}$ & $\begin{array}{l}\text { churna } \\
\text { (Powder) } \\
\text { +Gomutra }\end{array}$ & & & \\
\hline khair & khadir & $\begin{array}{l}\text { Acacia catechu (L. f.) } \\
\text { Willd. (Leguminosae) }\end{array}$ & 14109 & $\begin{array}{l}\text { Stem } \\
\text { Bark }\end{array}$ & kalka (Paste) & $\begin{array}{l}\text { varnavikara / } \\
\text { krushnata } \\
\text { (Dark } \\
\text { complexion / } \\
\text { tanning) }\end{array}$ & As required & $\begin{array}{l}\text { Take one tea spoonful ( } 3 \mathrm{gm}) \text { powder of } \\
\text { stem bark of khadir. Add } 125 \mathrm{mg} \text { powder of } \\
\text { turmeric, and sufficient quantity of water to } \\
\text { make paste. Apply on face and keep for } 2 \mathrm{hr} \\
\text { and wash. Continue the same for a week. } \\
\text { (Agnivesh 2007) }\end{array}$ \\
\hline halad & haridra & $\begin{array}{l}\text { Curcuma longa L. } \\
\text { (Zingiberaceae) }\end{array}$ & 2816 & Rhizome & & & & \\
\hline khadir & khadir & $\begin{array}{l}\text { Acacia catechu (L.f.) } \\
\text { Willd. (Leguminosae) }\end{array}$ & 14109 & $\begin{array}{l}\text { Stem } \\
\text { Bark }\end{array}$ & kalka (Paste) & $\begin{array}{l}\text { yuvavanpitika } \\
\text { (pimples) }\end{array}$ & As required & $\begin{array}{l}\text { Take one tea spoonful (3gm) powder of } \\
\text { stem bark of khadir, add } 125 \mathrm{mg} \text { powder of } \\
\text { turmeric and (half teaspoonful) powder of } \\
\text { thorns of shalmali. Add sufficient quantity } \\
\text { of water to make paste. Apply on face and } \\
\text { keep for } 2 \mathrm{hr} \text { and wash. Continue the same } \\
\text { for a week. (Sharma 2013) }\end{array}$ \\
\hline halad & haridra & $\begin{array}{l}\text { Curcuma longa L. } \\
\text { (Zingiberaceae) }\end{array}$ & 2816 & Rhizome & & & & \\
\hline
\end{tabular}




\begin{tabular}{|c|c|c|c|c|c|c|c|c|}
\hline kate savar & shalmali & $\begin{array}{l}\text { Bombax ceiba L. } \\
\text { (Bombacaceae) }\end{array}$ & 14052 & Thorns & & & & \\
\hline ambehalad & $\begin{array}{l}\text { aamragandhi } \\
\text { haridra }\end{array}$ & $\begin{array}{l}\text { Curcuma amada } \\
\text { Roxb. } \\
\text { (Zingiberaceae) }\end{array}$ & 14607 & Rhizome & $\begin{array}{l}\text { swarasa } \\
\text { (Juice) }\end{array}$ & $\begin{array}{l}\text { shotha } \\
\text { (Swelling) }\end{array}$ & $30 \mathrm{ml}$ & $\begin{array}{l}\text { Prepare the powder of ambehalad and stem } \\
\text { bark of bivala. Mix one table spoonful of } \\
\text { both ambehalad and bivala in the bulk of } 2 \\
\text { eggs. Administer the mixture orally, once a } \\
\text { day in early morning on empty stomach for } \\
4 \text { days. (Vaidya 1999, Nadkarni 1999) }\end{array}$ \\
\hline bivala & asana & $\begin{array}{l}\text { Pterocarpus } \\
\text { marsupium Roxb. } \\
\text { (Lequminosae) }\end{array}$ & 14288 & $\begin{array}{l}\text { Stem } \\
\text { bark }\end{array}$ & & & & \\
\hline mehandi & madayntika & $\begin{array}{l}\text { Lawsonia inermis L. } \\
\text { (Lythraceae) }\end{array}$ & 14357 & Leaf & $\begin{array}{l}\text { swarasa } \\
\text { (Juice) }\end{array}$ & $\begin{array}{l}\text { kamala } \\
\text { (Jaundice) }\end{array}$ & $30 \mathrm{ml}$ & $\begin{array}{l}\text { Extract the juice of } 7-8 \text { fresh leaves and add } \\
\text { the powder of } 1 \text { betel nut in the juice and } \\
\text { mix it in } 500 \text { ml cow's milk. Administer orally } \\
\text { twice a day on empty stomach for 2-3 days. } \\
\text { (Vaidya 1999, Sharma 2001) }\end{array}$ \\
\hline supari & puga & $\begin{array}{l}\text { Areca catechu L. } \\
\text { (Arecaceae) }\end{array}$ & 8850 & Fruit & & & & \\
\hline tanvel & rajapatha & $\begin{array}{l}\text { Cyclea peltata (Lam.) } \\
\text { Hook.f. \& Thomson } \\
\text { (Menispermiaceae) }\end{array}$ & 14629 & Root & $\begin{array}{l}\text { hima (cold } \\
\text { infusion) }\end{array}$ & $\begin{array}{l}\text { atisara } \\
\text { (Diarrhoea) }\end{array}$ & $30 \mathrm{ml}$ & $\begin{array}{l}\text { Prepare the fine powder of both ingredients } \\
\text { in equal quantity. Soak 3gm powder in 30ml } \\
\text { water overnight. Filter and administer the } \\
\text { cold infusion once a day for } 2 \text { days } \\
\text { (Bhavmishra 2006, Sharma 1998) }\end{array}$ \\
\hline saga & shaka & $\begin{array}{l}\text { Tectona grandis L.f. } \\
\text { (Lamiaceae) }\end{array}$ & 14046 & $\begin{array}{l}\text { Stem } \\
\text { Bark }\end{array}$ & & & & \\
\hline adulsa & vasa & $\begin{array}{l}\text { Justicia adhatoda L. } \\
\text { (Acanthaceae) }\end{array}$ & 3869 & Leaf & $\begin{array}{l}\text { kwatha } \\
\text { (Decoction) }\end{array}$ & kasa (Cough) & $30 \mathrm{ml}$ & $\begin{array}{l}\text { Take 4-5 leaves of adulsa and add 4-5 dried } \\
\text { fruits of miri and add in } 250 \mathrm{ml} \text { of water, } \\
\text { boil on low flame and reduce it to half the } \\
\text { quantity. Filter and administer } 30 \mathrm{ml} \\
\text { decoction twice a day for } 2 \text { days. (Sharma } \\
\text { 2013) }\end{array}$ \\
\hline miri & $\begin{array}{l}\text { maricha - } \\
\text { 10gm }\end{array}$ & $\begin{array}{l}\text { Piper nigrum L. } \\
\text { (Pipercaeae) }\end{array}$ & 672 & Fruit & & & & \\
\hline dhane & dhnayak & $\begin{array}{l}\text { Coriandrum sativum } \\
\text { L. (Apiaceae) }\end{array}$ & 1121 & Seed & $\begin{array}{l}\text { hima (cold } \\
\text { infusion) }\end{array}$ & $\begin{array}{l}\text { mutrashmari } \\
\text { (Urolithiasis) }\end{array}$ & $200 \mathrm{ml}$ & $\begin{array}{l}\text { Soak approx. } 50 \mathrm{gm} \text { seeds of coriander and } \\
10-12 \text { dried flowers of palash in } 200 \mathrm{ml} \\
\text { water, keep for overnight. Filter it in the next } \\
\text { morning. Administer filtrate orally once in } \\
\text { day for } 10 \text { days on empty stomach. } \\
\text { (Bhavmishra 2006) }\end{array}$ \\
\hline
\end{tabular}


Ethnobotany Research and Applications

\begin{tabular}{|c|c|c|c|c|c|c|c|c|}
\hline palas & palash & $\begin{array}{l}\text { Butea monosperma } \\
\text { (Lam.) Taub. } \\
\text { (Leguminosae) }\end{array}$ & 14185 & Flower & & & & \\
\hline gulvela & guduchi & $\begin{array}{l}\text { Tinospora cordifolia } \\
\text { (Willd.) Miers } \\
\text { (Menispermaceae) }\end{array}$ & 14207 & Stem & $\begin{array}{l}\text { dravan } \\
\text { (solution) }\end{array}$ & $\begin{array}{l}\text { madhumeha } \\
\text { (Diabetes) }\end{array}$ & $50 \mathrm{ml}$ & $\begin{array}{l}\text { Dissolve } 1 \mathrm{gm} \text { resin of asana in approx } 50 \mathrm{~m} \\
\text { water, till the water becomes reddish, later } \\
\text { mix approx. } 3 \text { gm powder of dried guduchi } \\
\text { stem. Administer orally twice a day for } 8 \\
\text { days. (Sharma 2013) }\end{array}$ \\
\hline bivala & vijayasar & $\begin{array}{l}\text { Pterocarpus } \\
\text { marsupium Roxb. } \\
\text { (Leguminosae) }\end{array}$ & 14288 & Resin & & & & \\
\hline
\end{tabular}




\section{Ethnomedicinal claims}

During the survey 182 folk claims were collected, amongst them, 172 were of single drug claim reported from 99 plant species and 10 numbers were of compound formulations of 16 plant species. This indicates the precise knowledge about the usage of particular medicinal plants in particular disease (Table. $2 . \& 3$. )

\section{Taxonomic Variation}

Data analysis showed that information on 100 drugs consisting of 99 plant species and 01 mineral drug were reported during the survey. Total 88 genera and 48 families are being used by the tribes for managing various ailments. The plant family with the highest number of plants cited were Leguminosae (29.17\%) followed by Apocynaceae (16.67\%) and Combretaceae (12.50\%) and Lamiaceae (10.42\%). (Fig. 2.)

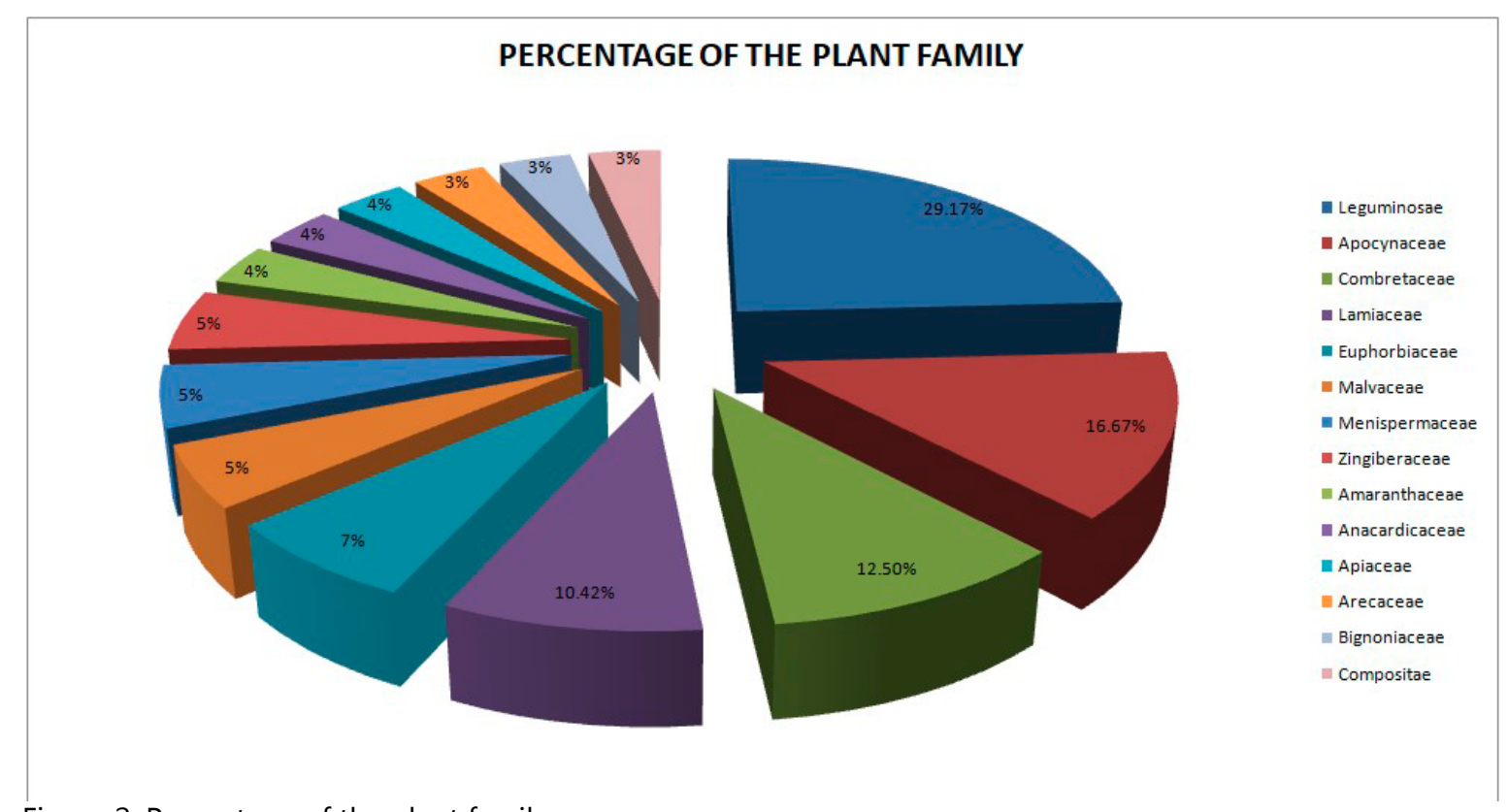

Figure 2. Percentage of the plant family

\section{The life form}

The data of the survey study revealed that amongst the different life form pattern, tress were used maximum 54\% for therapeutic purpose followed by herb and climber, as shown in Fig. 3. Similar result was obtained in previous study (Roy \& Janbandhu 2020).

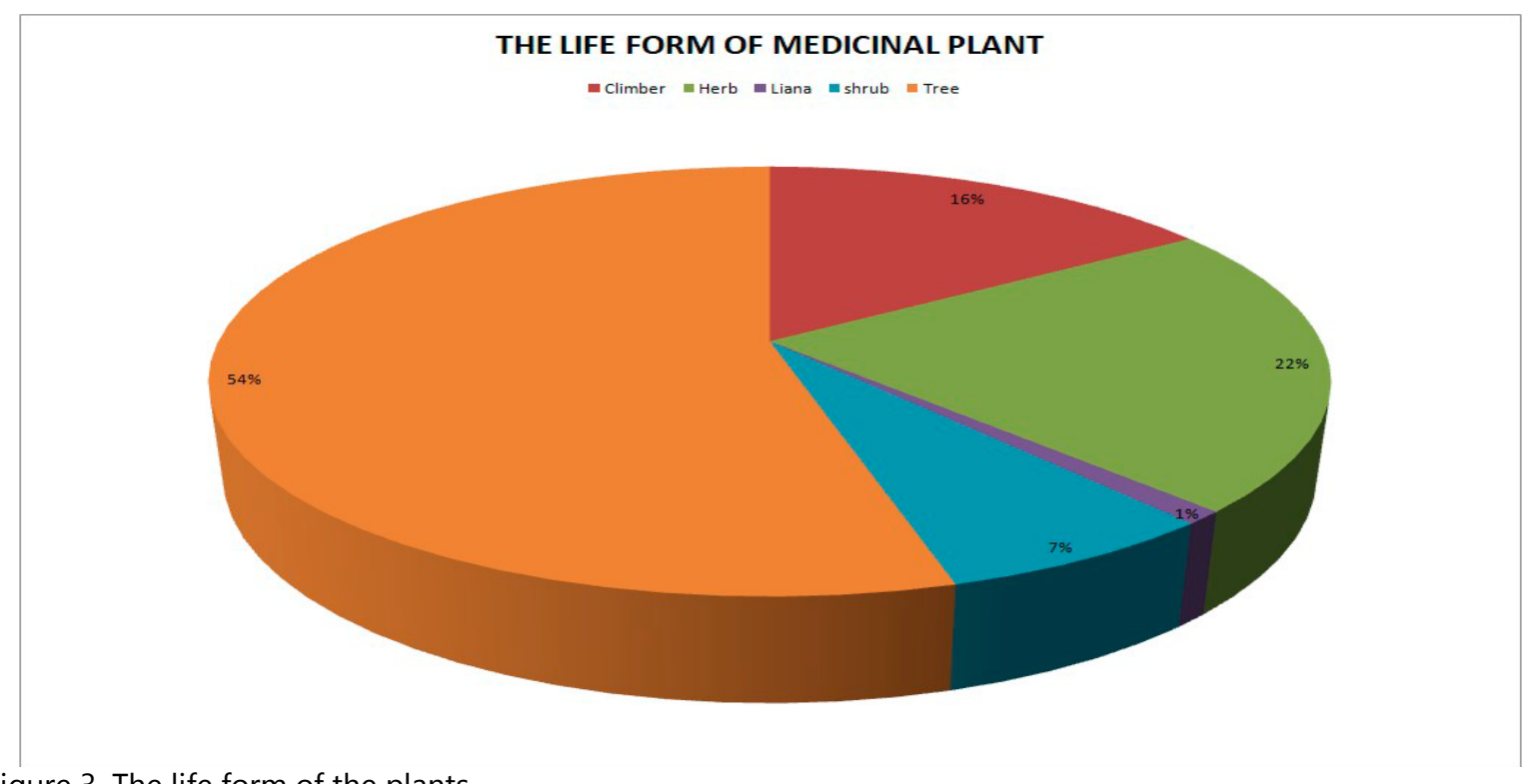

Figure 3. The life form of the plants 


\section{Plant part(s) used}

Amongst the 15 classes of the different useful parts of the plants, the stem bark was utilized the most (22\%) among the 99 plant species, followed by root (18\%) and leaf (18\%). Details are shown in Fig. 4.

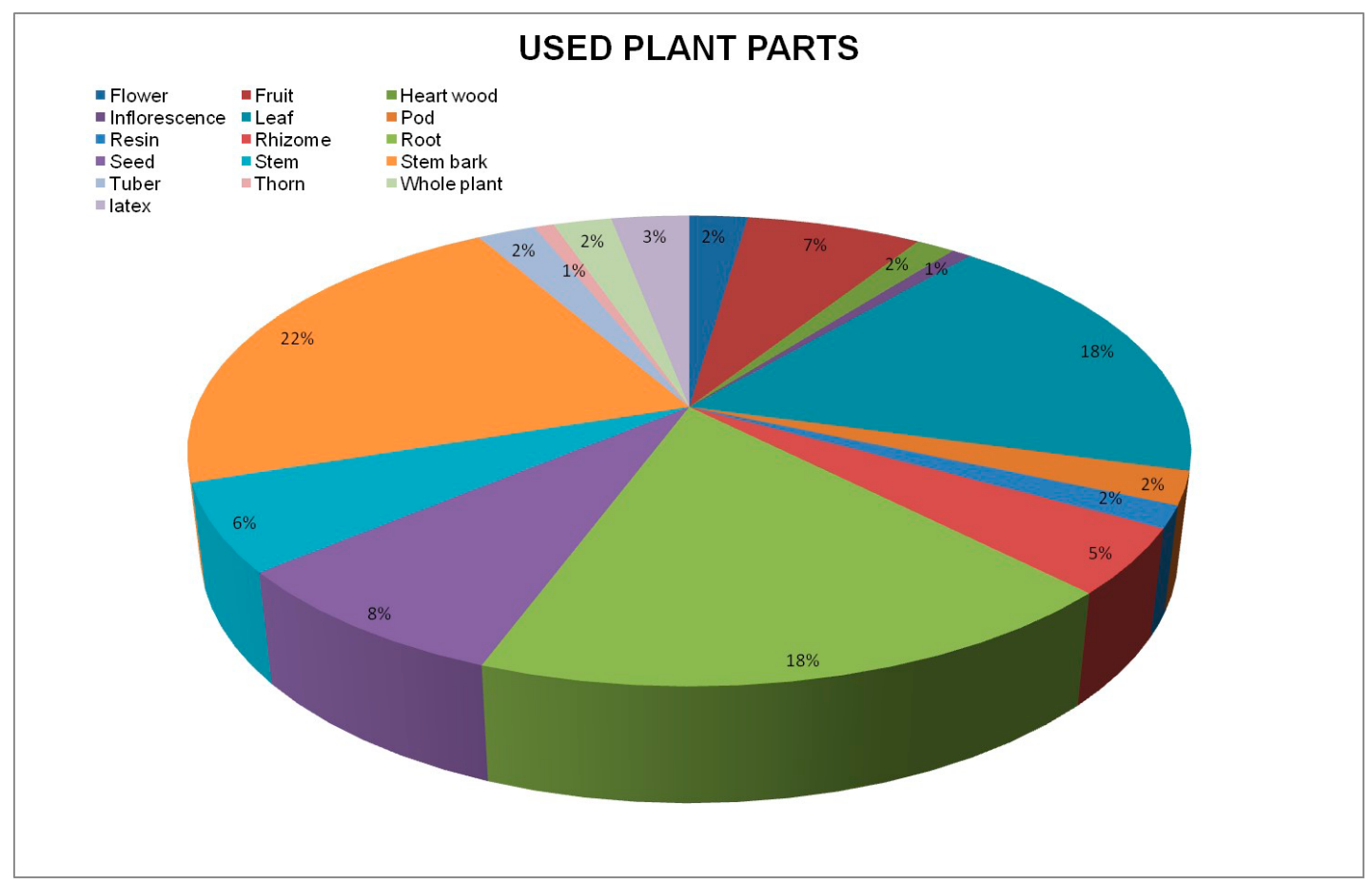

Figure 4. Used plant parts

\section{Form of formulations}

For the treatment of various ailments, respondents in the study region use various means for administering medicine, such as juice, decoction, paste, powder, medicated oil, paste, infusion, and ash. In studied area juice (swarasa) is used frequently (26.81\%), followed by paste (kalka) (19.55\%), and powder (churna) (13.40\%). Solution (dravana) is also used frequently (5.02\%) which is prepared by soaking powder in the water for 5-20 minutes. Other than this, pills (vati), hot infusion (phant ), cold infusion ( hima ), decoction (kwatha), ash ( mashi ), alkali (kshara), etc. forms of administration are used by the tribal. (Fig.5). In many folk claims, useful plant parts like root, stem, bark, leaf, fruit, the rhizome were advised to masticate in the fresh form, and aerial part, the latex of certain medicinal plants were used in the form of local application (21.22\%).

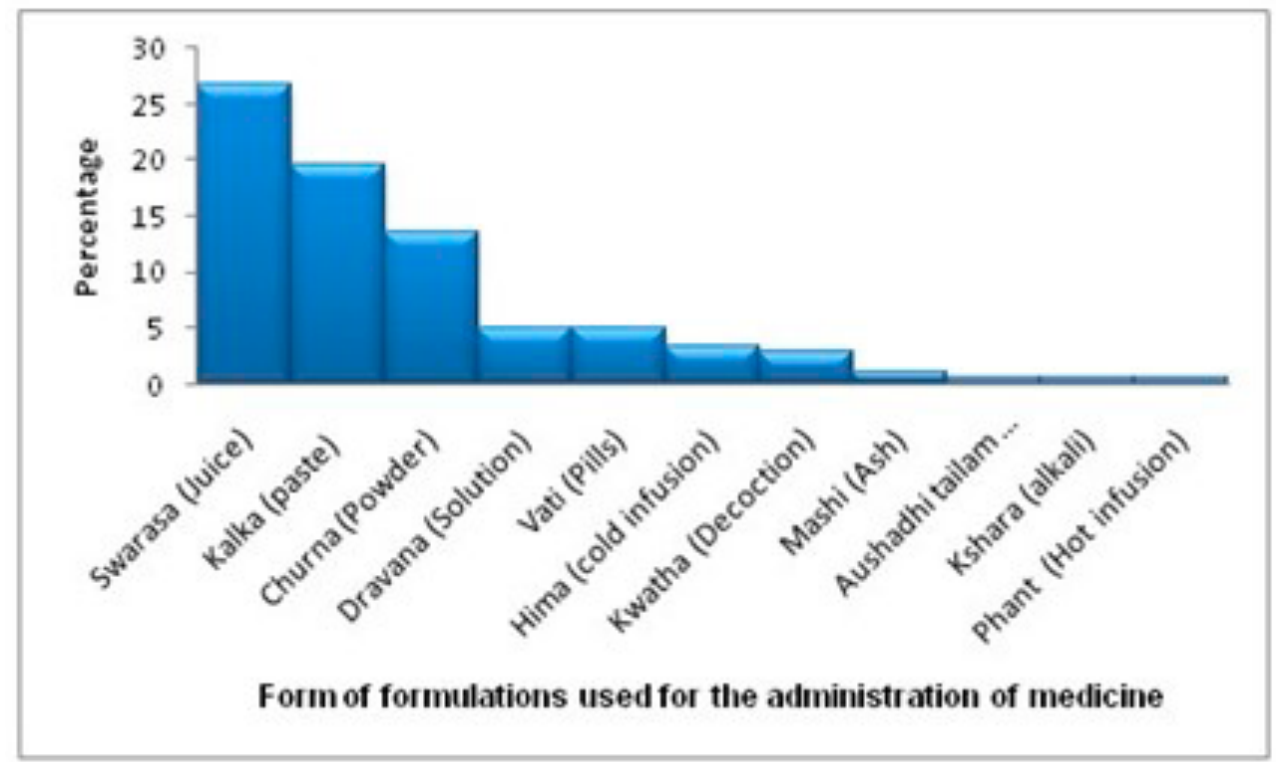

Figure 5 . The form of formulations used for the administration of medicines 


\section{Mode of administration}

Amongst the collected claims, $73.23 \%$ of remedies were administered orally whereas $26.77 \%$ were applied topically on the affected area supporting the previous studies (Roy \& Janbandhu 2020). Tribal also administers medicines in the form of dhumapana- (Medicated smoking) which was recorded 05 times, nasya- (Medication through nasal route) noted 03 times, karnapurana- (filling of the ear with medicated liquid) recorded once. For certain disease conditions like fever and jaundice, a bath of medicated water was also recommended 03 times. dhupana(Fumigation) and aschotana- (eye drops) is recorded twice and pradhamannasya- insufflations of medicated powder in the nose is recorded once. Moreover, the root of shatavari (Asparagus racemosus Willd), the twig of gambhari (Gmelina arborea L.) are also used as a necklace, and the whole plant of Cyclea peltata (Lam) Hook and Cissampelos pareira L. are used as a bracelet to treat the disease.

\section{Qualitative analysis}

\section{Informant Consensus Factor ICF}

To calculate ICF, the reported ailments were first classified into 06 different disease categories and Migraine was taken as a symptom. Among these, the highest ICF value of hypogalactia (0.67), followed by ICF of poisonous bite (0.36) were recorded as significant. Details of the calculated ICF values are exhibited in Fig. 6 and Table.4.

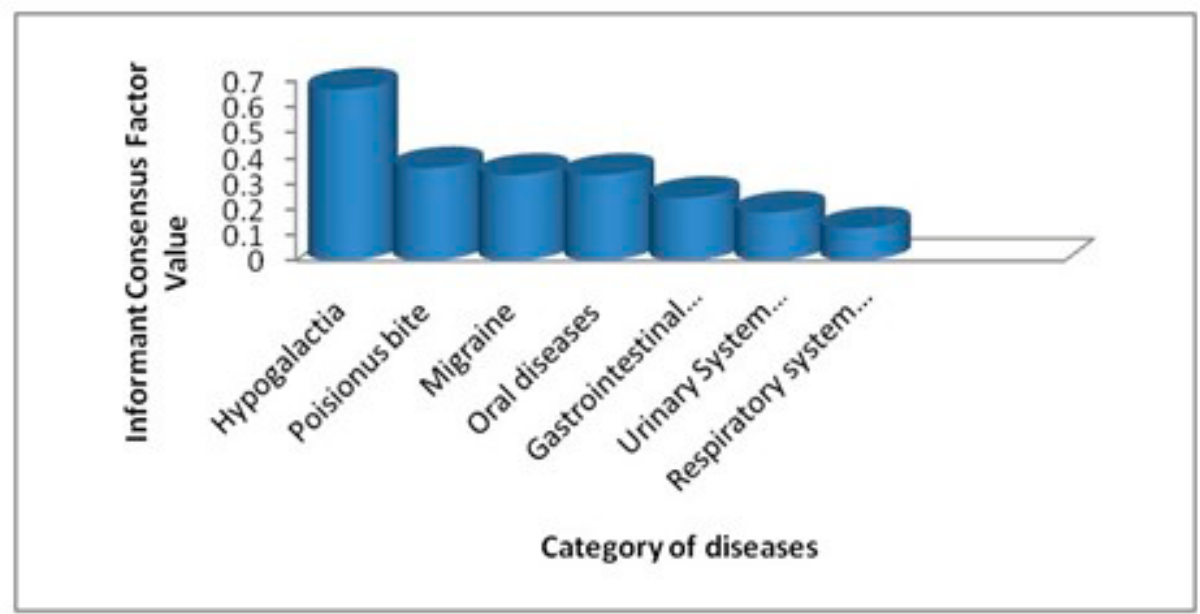

Table 4. Informant Consensus Factor of documented disease category during the survey

\begin{tabular}{llllll}
\hline Category of diseases & $\begin{array}{l}\text { Nur- (Number of } \\
\text { use citation) }\end{array}$ & $\begin{array}{l}\mathbf{N}_{\mathbf{t}} \text { (Number of } \\
\text { used species) }\end{array}$ & $\mathbf{N u r}_{\mathbf{n}} \mathbf{\mathbf { N } _ { \mathbf { t } }}$ & $\mathbf{N}_{\mathbf{u r}-\mathbf{1}}$ & ICF \\
\hline Gastrointestinal disease & 42 & 32 & 10 & 41 & 0.24 \\
\hline Oral disease & 4 & 3 & 1 & 3 & 0.33 \\
\hline Respiratory & 9 & 8 & 1 & 8 & 0.12 \\
\hline Poisonous bite & 26 & 17 & 9 & 25 & $\mathbf{0 . 3 6}$ \\
\hline Urinary & 17 & 14 & 3 & 16 & 0.19 \\
\hline Hypogalactia & 4 & 2 & 2 & 3 & $\mathbf{0 . 6 7}$ \\
\hline Migraine & 4 & 3 & 1 & 3 & 0.33 \\
\hline
\end{tabular}

Data indicated the significance of Informant consensus factor value on some categories of diseases gathered during the survey, whereas infectious disease, dental problem, gynecological disorders, skin diseases, metabolic disorders, musculoskeletal disorders, infertility, and symptoms like fever and wound showed 0 ICF value.

\section{Use Value Index}

Data analysis of plant species used or narrated by the respondents is expressed as Use Value (UV). The highest use value (UV) 0.13 was recorded for Holarrhena pubescens Wall. ex G. Don and Tinospora cordifolia (Willd.) Miers Details of the Use Value Index are shown in Fig. 7.

\section{Family Importance Value}

During the survey from Shahapur and Jawhar forest area, information on plant drugs of a total of 23 families was obtained from informers and folk healers. Among this maximum of $37.78 \%$ of utilization of plant, drugs were recorded of Leguminosae family, utilized by total 17 informants. Apocynaceae was recorded showing $28.89 \%$ 
importance value followed by Lamiaceae with $26.67 \%$ FIV. The family importance value of Euphorbiaceae and Menispermiaceae was recorded at $20 \%$ each. The details of the result of the FIV value is shown graphically (Fig. 8.)

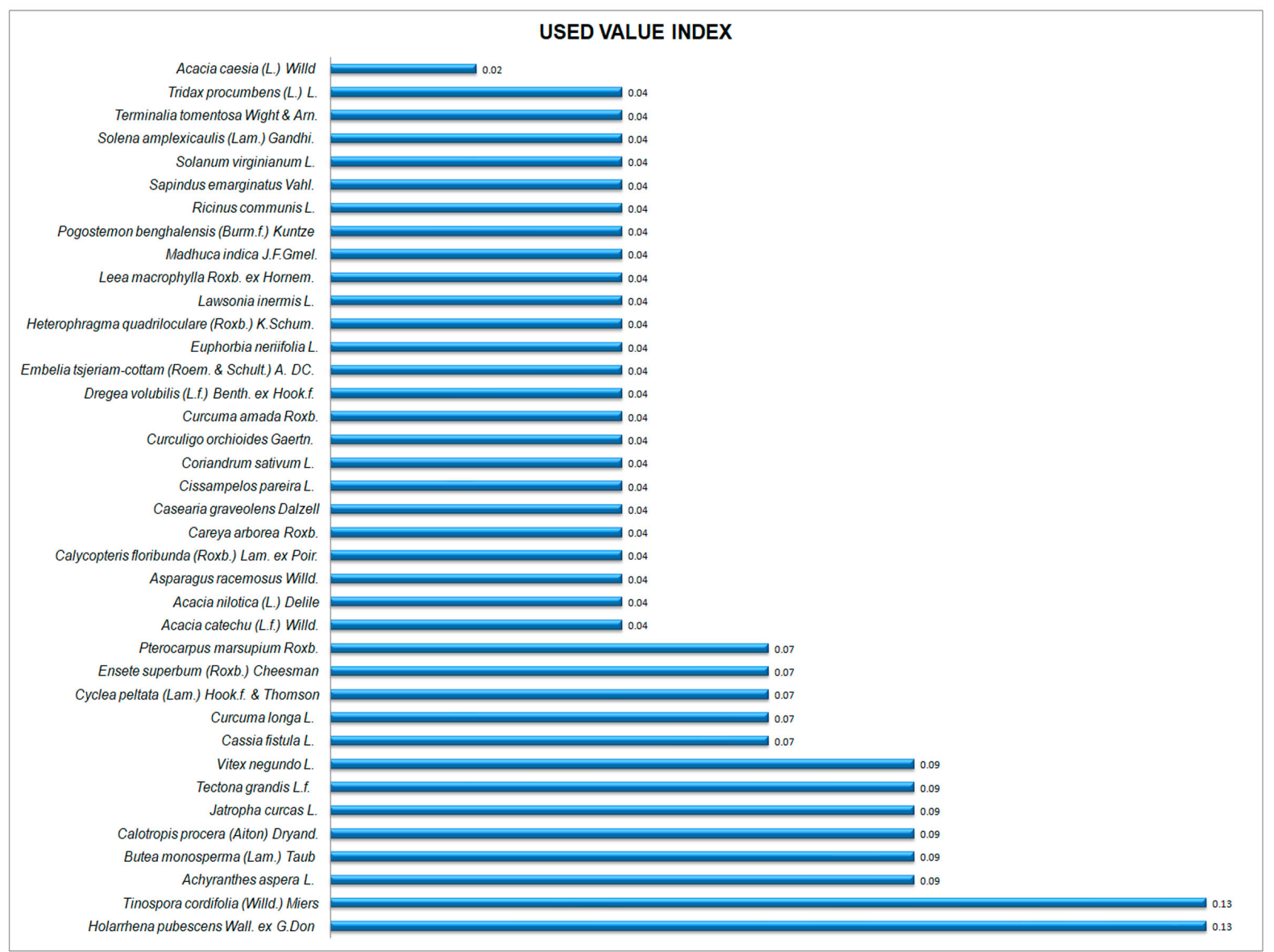

Figure 7. Use Value Index of plants

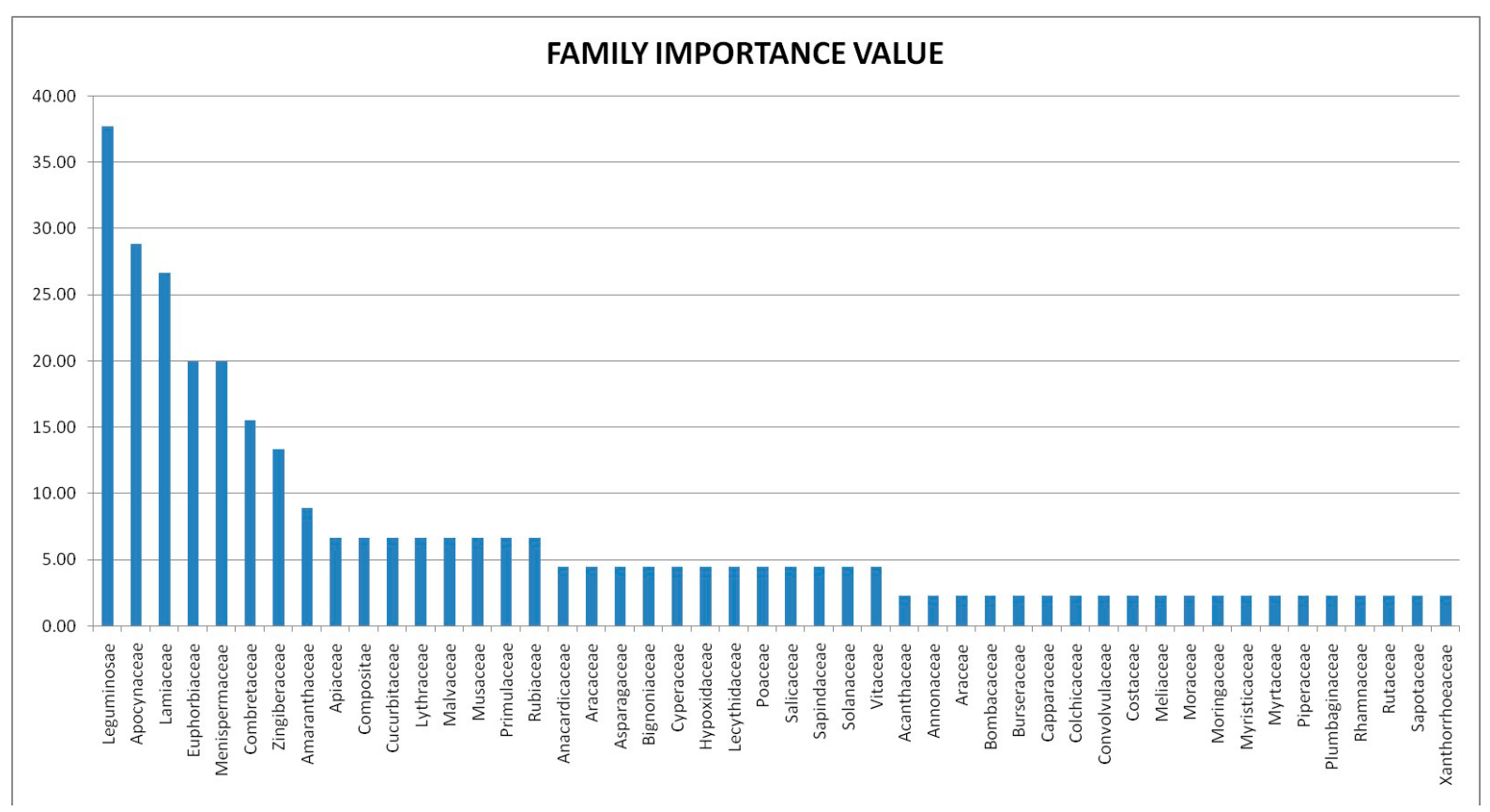

Figure 8. Family Importance Value 
Fidelity level is useful for determining which species are favoured by key informants for treating specific diseases. The FL values of frequently used medicinal plants that are by the locals are higher than those that are less widely used. The percentage of informants that claim to utilise a specific plant species for the same principal purpose is referred to as the fidelity level. The low value of FL indicates that plant species are used for several medicinal purposes and confirms that they are used infrequently against a specific ailment by the informants in the study area.

The medicinal plants that are widely used by the local people have higher FL values than those that are less popular. The present study revealed that 12 plant species are commonly used having more than 50\% FL for the most prevalent 10 diseases reported in the survey area. Cissampelos pareira L., Curcuma amada Roxb., Lawsonia inermis L. Casearia graveolens Dalzell, Dregea volubilis (L.f.) Benth. ex Hook. f., Heterophragma quadriloculare (Roxb.) K. Schum. Coriandrum sativum L. and Terminalia tomentosa Wight \& Arn. showed $100 \%$. Thus, native plants having medicinal values are used to combat diseases. Fidelity level (FL) in the study area showed its acceptance as a medicinal herb for a particular ailment category. Details of the result are showed in Table.5. and Fig. 9.

Table. 5. Fidelity level of medicinal plant in Thane forest circle, Maharashtra, India

\begin{tabular}{|c|c|c|c|c|}
\hline Botanical Name & Sanskrit Name & $\begin{array}{l}\text { Disease } \\
\text { categories }\end{array}$ & Specified Disease name & $\begin{array}{l}\text { Fidelity } \\
\text { level (\%) }\end{array}$ \\
\hline Cissampelos pareira L. & patha & $\begin{array}{l}\text { Gastrolntestinal } \\
\text { Tract Diseases }\end{array}$ & amatisara & 100.00 \\
\hline $\begin{array}{l}\text { Cyclea peltata (Lam.) } \\
\text { Hook. f. \& Thomson }\end{array}$ & rajpatha & & $\begin{array}{l}\text { atisara (Diarrhoea in } \\
\text { children) }\end{array}$ & 66.67 \\
\hline $\begin{array}{l}\text { Holarrhena pubescens } \\
\text { Wall. ex G. Don }\end{array}$ & kutaja & & Udarshula & 28.57 \\
\hline Curcuma amada Roxb. & $\begin{array}{l}\text { aamragandhi } \\
\text { haridra }\end{array}$ & $\begin{array}{l}\text { Inflammatory } \\
\text { conditions }\end{array}$ & $\begin{array}{l}\text { aghataj shotha, } \\
\text { (traumatic swelling) }\end{array}$ & 100.00 \\
\hline Lawsonia inermis L. & madayntika & Liver disorder & kamala (Jaundice) & 100.00 \\
\hline $\begin{array}{l}\text { Calotropis procera } \\
\text { (Aiton) Dryand. }\end{array}$ & arka & $\begin{array}{l}\text { Neurological } \\
\text { disease }\end{array}$ & $\begin{array}{l}\text { ardhavabhedak } \\
\text { (Migraine) }\end{array}$ & 50.00 \\
\hline Achyranthes aspera L. & apamarga & Poisonous bite & $\begin{array}{l}\text { vruschik dansha } \\
\text { (scorpion sting) }\end{array}$ & 50.00 \\
\hline $\begin{array}{l}\text { Casearia graveolens } \\
\text { Dalzell }\end{array}$ & - & & Sarpadansha (Snake bite) & 100.00 \\
\hline $\begin{array}{l}\text { Dregea volubilis (L. f.) } \\
\text { Benth. ex Hook. f. }\end{array}$ & brhatpurva & & sarpadansha (Snake bite) & 100.00 \\
\hline $\begin{array}{l}\text { Embelia tsjeriam- } \\
\text { cottam (Roem. \& } \\
\text { Schult.) A. DC. }\end{array}$ & vidang bhed & & sarpadansha (Snake bite) & 66.67 \\
\hline $\begin{array}{l}\text { Ensete superbum } \\
\text { (Roxb.) Cheesman }\end{array}$ & vanyakadali & & shwan dansha (Dog bite) & 66.67 \\
\hline Jatropha curcas L. & dravanti & & sarpadansha (snake bite) & 50.00 \\
\hline $\begin{array}{l}\text { Pogostemon } \\
\text { benghalensis (Burm. f.) } \\
\text { Kuntze }\end{array}$ & - & & $\begin{array}{l}\text { vrushchikdansha } \\
\text { (Scorpion sting) }\end{array}$ & 100.00 \\
\hline $\begin{array}{l}\text { Butea monosperma } \\
\text { (Lam.) Taub. }\end{array}$ & palash & $\begin{array}{l}\text { Reproductive } \\
\text { diseases }\end{array}$ & $\begin{array}{l}\text { aniyamit artava chakra } \\
\text { (Irregular menstrual cycle) }\end{array}$ & 50.00 \\
\hline $\begin{array}{l}\text { Heterophragma } \\
\text { quadriloculare (Roxb.) } \\
\text { K. Schum. }\end{array}$ & waras & $\begin{array}{l}\text { Dermatological } \\
\text { diseases }\end{array}$ & $\begin{array}{l}\text { chikhalya (M) alaji (Tinea } \\
\text { pedia) }\end{array}$ & 100.00 \\
\hline Coriandrum sativum L. & dhanyak & $\begin{array}{l}\text { Urinary Tract } \\
\text { Diseases }\end{array}$ & $\begin{array}{l}\text { mutrashmari } \\
\text { (urolithiasis/ renal calculi) }\end{array}$ & 100.00 \\
\hline Tectona grandis L. f. & shaka & & ashmari & 50.00 \\
\hline $\begin{array}{l}\text { Terminalia tomentosa } \\
\text { Wight \& Arn. }\end{array}$ & arjuna bheda & Wound & vrana & 100.00 \\
\hline Vitex negundo L. & nirgudi & Migraine & shirashula (Head ache) & 50.00 \\
\hline
\end{tabular}




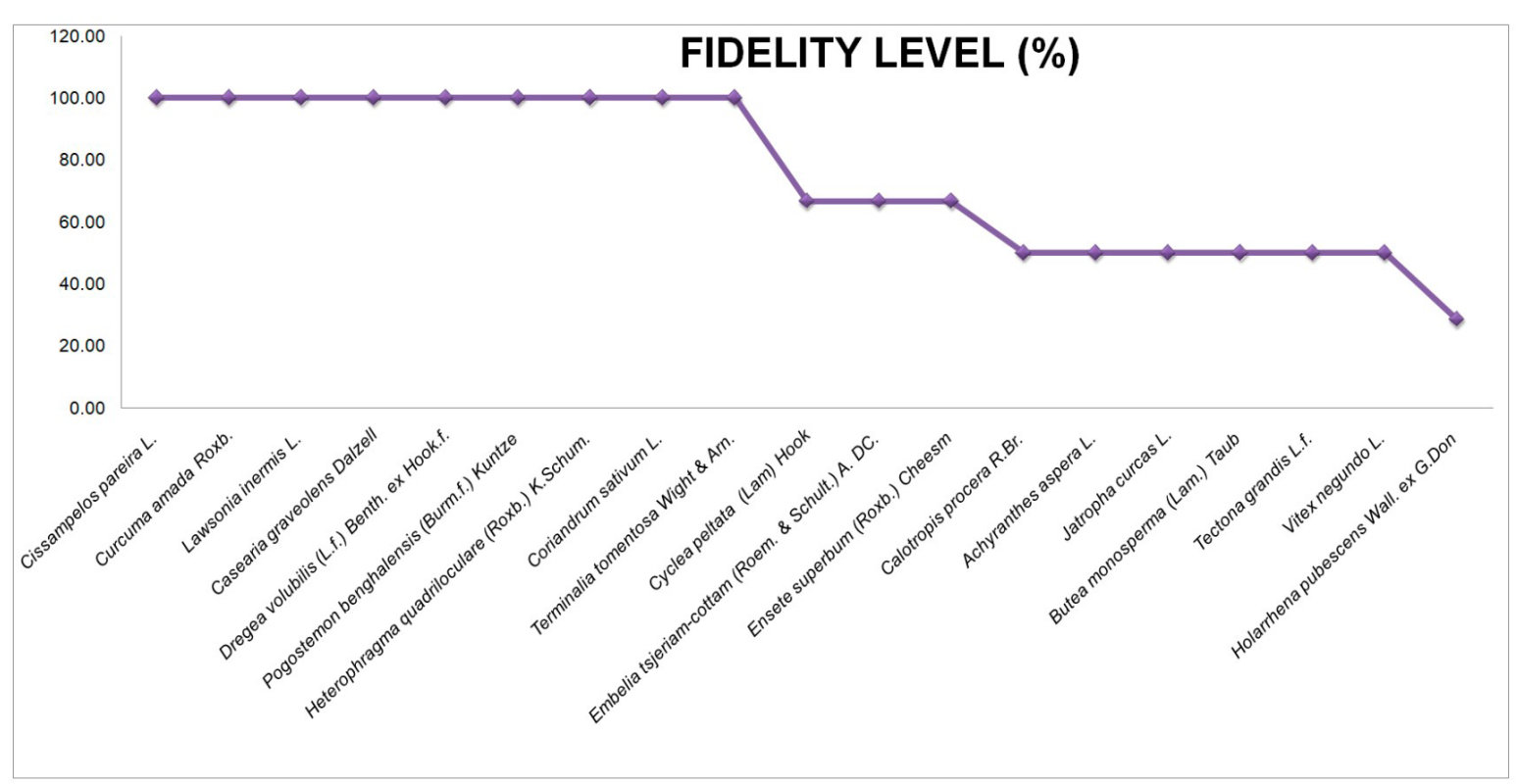

Figure 9. Fidelity level (\%)

\section{Discussion}

In the survey zone, the project team interacted with many local individuals who had basic information regarding traditional medicine but to get authentic and reliable information the data was collected only from the traditional folk healers known as vaidu / bhagat in local language and the respondents who were well versed and had complete information of the claims. It was observed that maximum information was shared by illiterate and elder informants on the traditional use of medicinal plant species as compared to educated informers. More dependency on allopathic doctors their availability in nearby areas and mode of transportation, less belief in folk healers, etc. are some of the reasons to decline interest of the young generation towards traditional health practice. Similar observations are also reported in other countries also (Farooq et al. 2019, Mafuva \& Marima-Matarira 2014) It was also noticed in previous ethnomedicinal studies that, native people who are illiterate are more habituated to using ethnomedicinal herbs than those who are literate in the same area and other parts of the world (Roy \& Janbandhu 2020, Tugume et al. 2016,). Tribal folk healers are mostly dependent on the traditional method of diagnosis which is well explored as the asthvidhapariksa (eight-fold examination) in the Ayurveda. Nadipariksha (Pulse examination) is the prime method of examination done by maximum folk healers (Panda \& Mishra 2010). It is used for assessing tridoshas and various physiological and psychological states of the patient in Ayurveda. (Kumar et al. 2019) udar pariksha (Abdominal examination) is done by touching and palpating the abdomen, (Fig. 10.), whereas the sclera of eyes, palm, and tongue are also checked for the diagnosis of anemia, jaundice, etc. Thus, the method of examination of has Ayurvedic foundations, but many of them have indigenous roots based on personal experience and oral tradition (Deshmukh \& Pardeshi 2014) Along with this, they also considered the diagnosis done by the Modern physician eg. Urinary stone, fracture.

Western ghat located in Thane forest circle is dominant in Leguminosae, Euphorbiaceae, family as plants of these family is reported maximum in the survey area. (Rao R. http://wgbis.ces.iisc.ernet.in/biodiversity/sahyadri)

Amongst the useful plants, the maximum used plants were of the tree followed by herb and climber found similar observation as per the previous study (Roy \& Janbhandu 2020). Stem bark was reported as maximum, followed by leaves as used part of medicinal plants, whereas in many of the previous study leaves are reported maximum as useful plant part (Roy \& Janbandhu 2020, Jeyaprakash et al. 2011). Many of the essential phytoconstituents are extracted from the bark. quinine is extracted from Cinchona bark (Pasztory et al. 2016).

In the survey study, folk claims for gastrointestinal disease are reported by maximum respondents (42), indicating the prevalence of disease in the study area. Lack of adequate sewage management and sanitation systems, defecation in open fields, drinking contaminated water, food, poor hygiene, etc may be the reason for more prevalence of Gastrointestinal related diseases. (Tripurari et al. https://www.cdc.gov ) Therefore most of the healers were well versed in its treatment. Similar results were reported in other survey studies. (Khan 2016), likewise Cases of the poisonous bite were also very common in tribal areas which is also supported by the previous report. As, 
farmers, plantation workers, herdsmen, hunters, or field workers are mostly affected by snakebite and scorpion sting, particularly in rainy and summer seasons (Warrell 1999). Many respondents also reported folk claims for urinary problems. As selected area faced drouth in the summer season, and risk of urolithiasis in people working outdoors or exposed to high temperatures, such as workers, farmers, laborers are twice (Chadrajith et al. 2006, Ganesamoni \& Singh 2012) as compared to persons working in the room temperature also support the findings. Local health practitioners use native plant resources to cure 50 ailments ranging from fever to diabetes. Jaundice, abdominal pain, urinary stones, piles, diabetes, joint pain, and poisonous bites are the most prevalent disorders in the surveyed area. Natural plants are being used by the tribal to treat them. It demonstrates that the indigenous people of this area have a thorough understanding of ailments, remedies, and the identification of plants.

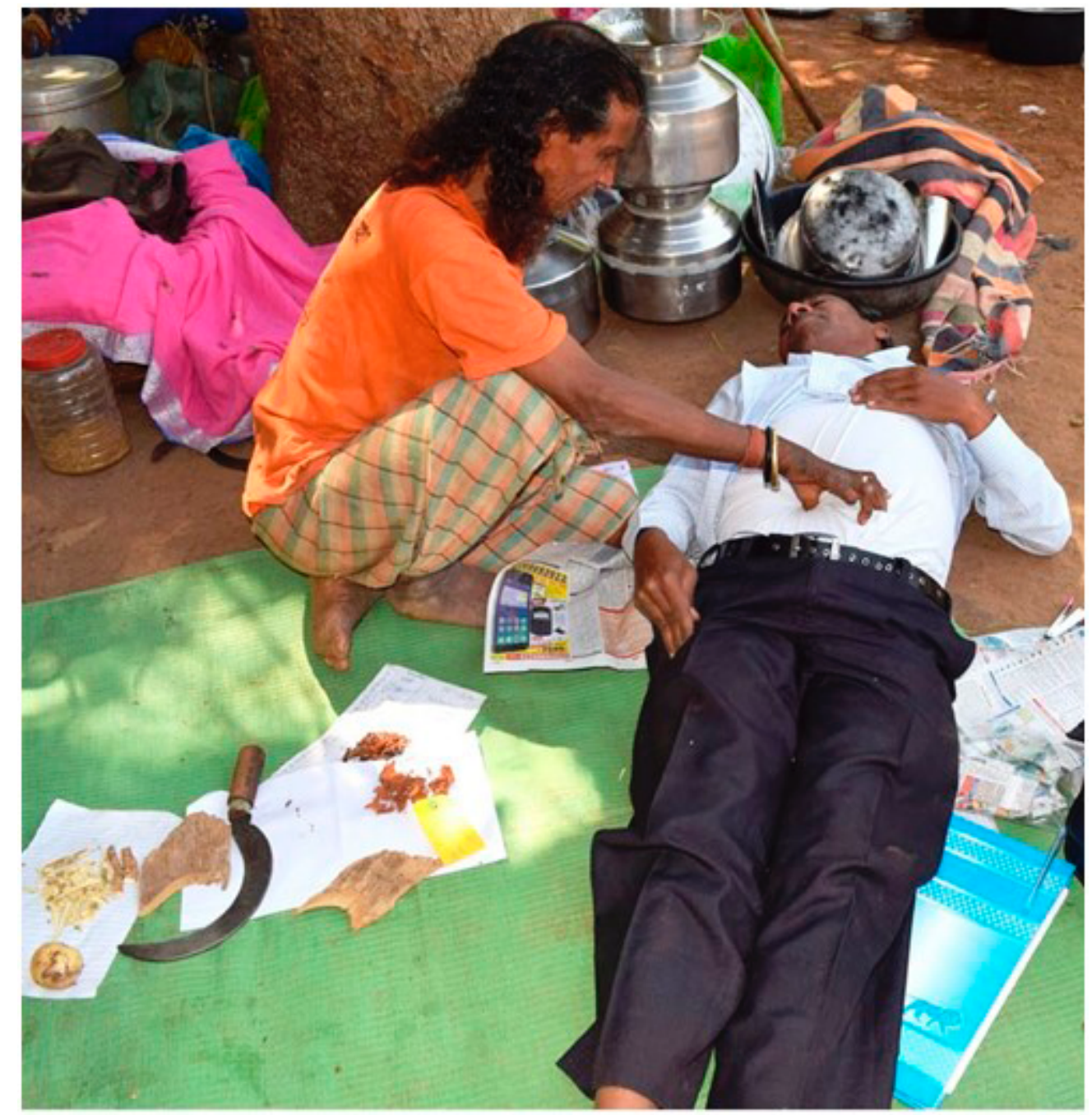

Figure.10. Folk healer doing the abdominal examination for the diagnosis of the disease

ICF value showed that limited plants species with unanimous opinion was recommended amongst different respondents. Early age pregnancy and malnourishment are the major problems faced by the tribal woman lead to hypogalactia (Ghosh \& Varerkar 2019).

After the diagnosis of disease, folk healers recommend the medicines best of their knowledge either in fresh form or prepare the combination of raw drugs and administer in required form like decoction, paste, etc. it was observed that tribal use all the major and subtype of form of administration depicted in the Ayurveda. Panchvidhakashayakalpana (Five basic preparations of medicines) includes Juice, paste, decoction, Hot and cold infusion. (Sharnagdhara 2008) are the major form of medicines used for the administration of medicines. The observations of this study are contrary to the previous study where Powder (churna) form is reported maximum in the Palghar area (Roy \& Janbandhu 2020). In the present study, Juice was reported maximum and is considered as the most potent form in the Ayurveda also. (Sharnagdhara 2008) Since the area of Shahapur and Jawhar receiving 
high rainfall as it is situated in the Western Ghats, bestowed with rich floristic flora, therefore, the availability, accessibility, and knowledge of indigenous plants make their choice to use fresh juice of useful plant part.

It was interesting to know that tribal is well versed about the different routes of administration of medicine other than oral and topical like Nasal application of medicine (nasya), smoking (dhumpana), etc. even proves the impact of Ayurveda. But other routes of administration like through vagina, rectal, intra-urethral were not reported in the studied area, unlike the description in Ayurveda (Pardeshi et al. 2016). Moreover, the use of well-practiced plants of Ayurveda as bracelets and necklaces showed the novelty of the studied area.

It is revealed that the use of Holarrhena pubescence (Buch.-Ham.) Wall ex DC. In diarrhea and dysentery was recorded with maximum UV, proves the immediate measure taken by the tribal by the easily available native plant. Kutaj (H. pubescence) is also considered the best drug for the treatment of diarrhea in Ayurveda. (Agnivesh 2007). The stem of Tinospora cordifolia (Willd.) Hook.F. \&Thoms., is preferably used for the treatment of diabetes, piles, fever as reported in the Ayurveda. (Agnivesh 2007). Thus, native plants are observed with maximum UV. The use of plants by the tribal are reported for similar therapeutic use mentioned in the traditional medicinal system like Ayurveda, Unani reported in other studies also. (Uniyal et al. 2006)

FIV value showed that Leguminosae, Apocynaceae, Lamiaceae are the most recorded family in the study area. Leguminosae is also the dominant family in India (Jain 1983) and the largest family of ethnopharmacological importance (Macido et al. 2018). In Bangladesh also plants belonging to the Leguminosae family are reported with maximum medicinal uses (Rehman \& Parvin 2014)

Plants that reported higher FL values are frequently used in the study region, showed the specific importance for a specific purpose. In the study are FL ranged from 28.57 to 100\%. Amongst the medicinal plants reported with 100 \%. FL viz., Cissampelos pareira L., Curcuma amada Roxb., Lawsonia inermis L. and Coriandrum sativum L. are also well-established plants in Ayurveda for similar therapeutic purposes as mentioned by tribal. Even the plants with low fidelity levels may be due to differences in the learning experience, tradition, and availability.

\section{Limitation}

The ethnomedicinal study is intended to record the traditional health practice adopted by the tribal, leaving in the remote area, close to nature. Their primary source of management of any ailment is a natural source available in their native area, but they didn't keep a record of such ancestral knowledge however share such wisdom by oral communication only. During this survey study, there were few well-known folk healers who refused to share any information due to linguistic obstacles and apprehension. Respondents other than folk healers were aware of such information but had incomplete information or not well versed for identification of authentic plants and disease condition.

\section{Novelty and future impact}

After the validation of reported claims, it was observed that 16 claims of single drug and all compound formulations are not recorded in the classical textbooks of Ayurveda. Ayurveda is a treasure house of vast knowledge recorded in numbers of classical literature and to validate each claim from all literature is quite difficult. Hence for present study important classical textbooks and authorized textbooks having compilation of references were referred. Amongst the 16 single drug claims, 14 plants are mentioned in the Ayurveda whereas fangali- Pogostemon benghalensis (Burm.f.) Kuntze. and kalihalad- Curcuma caesia Roxb. these two plant species are novel and could be explored further to screen bioactive compounds and their pharmacological activities to introduce in Ayurvedic Pharmacopoeia. Plants mentioned in the compound formulations are reported in Ayurveda, but the combination of plants and its indications reported in survey area are novel. The present study is unique in terms of documentation of details about the medicinal plants; its method of preparation, mode of application, and indication reported by the survey team consisting of Ayurveda Physician and Botanist. It helps to minimize the ambiguity regarding symptoms, method of diagnosis, an indication as well as botanical identification.

\section{Conclusion}

After the validation of reported claims, it was observed that 16 claims of a single drug and all compound formulations are not recorded in the classical textbooks of Ayurveda. Ayurveda is a treasure house of vast knowledge recorded in numbers of classical literature and to validate each claim from all literature is quite difficult. Hence for the present study important classical textbooks and authorized textbooks having a compilation of references were referred. Amongst the 16 single drug claims, 14 plants are mentioned in the Ayurveda whereas 
fangali- Pogostemon benghalensis (Burm.f.) Kuntze. and kali halad- Curcuma caesia Roxb. these two plant species are novel and could be explored further to screen bioactive compounds and their pharmacological activities to introduce in Ayurvedic Pharmacopoeia. Plants mentioned in the compound formulations are reported in Ayurveda, but the combination of plants and its indications reported in the survey area are novel. The present study is unique in terms of documentation of details about the medicinal plants; their method of preparation, mode of application, and indication reported by the survey team consisting of Ayurveda physicians and botanists. It helps to minimize the ambiguity regarding symptoms, method of diagnosis, an indication as well as botanical identification.

\section{Declarations}

List of abbreviation: UV: Use Value; ICF: Informant Consensus Factor; FIV: Family Importance value (FIV); FL: Fidelity level; LHT: Local Health Tradition; FRLHT: Foundation for Revitalisation of Local Health Traditions

Ethics approval and consent to participate: All the participants provided prior informed consent before the interviews.

Availability of data and materials: Data are available from the first author and corresponding Author.

Competing interests: The authors declare that they have no competing interests.

Funding: Funding for this project received from Central Council for Research In Ayurvedic Science, Ministry of AYUSH, Government of India

Author contributions: RK, AG and GP carried out the field study and wrote the manuscript. AG, CR and AM contributed in specimen identification. NS thoroughly revised the manuscript. All authors read and approved the manuscript.

\section{Acknowledgments}

The authors are thankful to the Director-General Central Council of Research in Ayurvedic Science for his valuable guidance and support. The authors also express their gratitude to the Chief Conservator of Forest Thane Forest circle and Deputy Conservator of Forest Jawhar and Shahapur area, for providing administrative permission, cooperation, and support for the survey. All the informants of the survey study are cordially acknowledged for their valuable cooperation throughout the survey study.

\section{Literature cited}

Agnivesh. 2007. Charaka Samhita with 'Ayurveda-Deepika' Commentary of Chakrapanidatta. Chowkhamba Sanskrit Sansthana, Varanasi, India.

Ali A, Akhtar N, Khan BA, Khan MS, Rasul A, Khalid N. 2012. Acacia nilotica. a plant of multipurpose medicinal uses. Journal of Medicinal Plants Research 6(9):1492-1496.

Anonymous. 1999. An Appraisal of Tribal Folk Medicines. Central Council for Research in Ayurvedic\& Siddha, New Delhi, India.

Anonymous. 1999. The Ayurvedic Pharmacopoeia of India. Department of ISM \& H, Ministry of Health and Family welfare, Govt. of India, New Delhi, India.

Anonymous. 2000. Ayurvedic formulary of India. Part.1-3 Department of ISM \& H, Ministry of Health and Family welfare, Govt. of India, New Delhi, India.

Anonymous.1986. CSIR: Medicinal and Aromatic Plants of India. Jammu- Tawi; Council of Scientific and Industrial Directorate, New Delhi, India.

AYUSH Ministry . 2014. AYUSH system . https://main.ayush.gov.in/ayush-systems (Accessed 30/03/2019)

Azaizeh H, Fulder S, Khalil K, Said O. 2003. Ethno Medicinal Knowledge of Local Area Practitioners in the Middle East Region. Fitoterapia 14:98-108.

Bhavmishra. 2006. Bhavprakash Nighantu, with KC Chunekar commentary. Chaukhambha Bharati Academy, Varanasi, India.

Billore KV, Yelne MB, Dennis TJ, Chaudhari BG. 2004. Database on Medicinal plants used in Ayurveda Vol-6. Central Council For Research in Ayurvedic\& Siddha, New Delhi, India.

Bioprospecting project. https://www.teriin.org/projects/nutrition-security/JSW-bio.php 
Brown CH. 1977. Folk Botanical Life-Forms: Their Universality and Growth. American Anthropologist 79:317-342.

Canales M, Hernández T, Caballero J, De Vivar AR, Avila G, Duran A. 2005. Informant consensus factor and antibacterial activity of the medicinal plants used by the people of San Rafael Coxcatlán, Puebla, México. Journal of Ethnopharmacology 97(3):429-439.

Chandrajith R, Wijewardana G, Dissanayake CB, Abeygunasekara A. 2006. Biomineralogy of human urinary calculi (kidney stones) from some geographic regions of Sri Lanka. Environment Geochemistry and Health 28:393-399.

Chaudhury R Roy \&MuchtarU.Traditional medicine in Asia. WHO 2001.

Chopra RN. 1956. Glossary of Indian medicinal plants. Council of Scientific \& Industrial Research, New Delhi, India.

Desai VG. 2015. Aaushadhi Sangraha (Marathi). Rajesh Publication, Pune, India.

Deshmukh RR, Pardeshi VN. 2014. Disease Diagnosis and treatment methods of herbalist in GautalaAutram-Ghat area of Marathwada, State Maharashtra. International Journal of Herbal Medicine 2(1):58-64.

Deshpamde AP, Javalgekar RR, Ranade S. 2018. Dravyagunavigyana Part I and II. Profishant Publisher, Pune, India.

Dey A, De JN. 2012. Traditional use of plants against snakebite in Indian subcontinent: a review of the recent literature. African Journal of Traditional, Complementary and Alternative Medicines 9(1):153-174.

Dey YN, Wanjari MM, Kumar D, Lomash V, Jadhav AD. 2016. Curative effect of Amorphophallus paeonïfolius tuber on experimental hemorrhoids in rats. Journal of ethnopharmacology 192(4):183-191.

Farooq A, Amjad MS, Ahmad K, Altaf M, Umair M, Abbasi AM. 2019. Ethnomedicinal knowledge of the rural communities of Dhirkot, Azad Jammu and Kashmir, Pakistan. Journal of Ethnobiology and Ethnomedicine 15(1):130.

Friedman J, Yaniv Z, Dafni A, Palewitch D. 1986. A preliminary classification of the healing potential of medicinal plants, based on a rational analysis of an ethnopharmacological field survey among Bedouins in the Negev Desert, Israel. Journal of Ethnopharmacol ogy16:275-287.

Ganesamoni R, Singh SK. 2012. Epidemiology of stone disease in northern India. In: Talati J, Tiselius HG, Albala DM, YE Z, editors. Urolithiasis: basic science and clinical practice. Vol-1, Epidemiology, Springer London, London, Pp. $39-46$.

Ghosh S, Varerkar SA. 2019. Undernutrition among tribal children in Palghar district, Maharashtra, India. PloS one 14(2):423-33.

Goswami A, Barooch PK, Sandhu JS. 2002. Prospect of herbal drugs in the age of globalization - Indian scenario. Journal of Science Research 61:423-443.

Hemadri K, Sharma PC, Narayanappa D, Sashibhusshan S, Murthy KS. 1996. Medico-Botanical exploration of Phulabani and Koraput District of Orissa. Central Council For Research in Ayurvedic\& Siddha, New Delhi, India.

http://cgwb.gov.in/District_Profile/Maharashtra/Thane.pdf(Accessed 17/08/2021)

http://www.theplantlist.org (Accessed 01/09/2020)

https://apps.who.int/iris/bitstream/handle/10665/206025/B0104.pdf;jsessionid=404102307D1AA68B21D45A1A80 336755? sequence $=1$ (Accessed 2/03/2020)

https://censusindia.gov.in/2011census/dchb/DCHB_A/27/2721_PART_A_DCHB_THANE.pdf (Accessed 20/08/2021) https://en.wikipedia.org/wiki/ (Accessed 11/07/2020)

https://gazetteers.maharashtra.gov.in/cultural.maharashtra.gov.in/english/gazetteer/land_and_people/L\%20\&\%2 OP\%20pdf/Chapter\%20II/2\%20Major\%20Castes\%20and\%20Tribes.pdf (Accessed 11/08/2021)

https://www.ethnobiology.net/what-we-do/core-programs/ise-ethics-program/code-of-ethics/,(Accessed 22/06/2020)

India Biodiversity Portal. https://indiabiodiversity.org/(Accessed 2/02/2021)

Jain SK. 1983. Flora and Vegetation of India-An outline Botanical Survey of India Dept. Environment, New Delhi. 
Jaiswal YS, Williams LL. 2017. A glimpse of Ayurveda-The forgotten history and principles of Indian traditional medicine. Journal of traditional and complementary medicine 7(1):50-53.

Jeyaprakash K, Ayyanar M, Geetha KN, Sekar T. 2011. Traditional uses of medicinal plants among the tribal people in Theni District (Western Ghats), Southern India. Asian Pacific Journal of Tropical Biomedicine 1(1):S20-5.

Kaunda JS, Zhang YJ. 2017. The genus Carissa: An ethnopharmacological, phytochemical and pharmacological review. Natural Products and Bioprospecting 7(2):181-199.

Khan Z. 2013. Ethnobotanical survey of Thane district. Annals Of Pharmacy And Pharmaceutical Science 4(2):22-25.

Kumar B, Suryanarayana. 2011. Promising antidote plant species from the tribals of Sriharikota Island, Andhra Pradesh. Life Sciences Leaflets 19:769-779.

Kumar PV, Deshpande S, Nagendra HR. 2019. Traditional practices and recent advances in NadiPariksha: a comprehensive review. Journal of Ayurveda and Integrative Medicine 10(4):308-315.

Lavekar GS, Chandra K, Dhar BP, Mangal AK, Dabur R, Gurav AM. 2007. Database on Medicinal plants used in Ayurveda Vol-8. Central Council For Research in Ayurvedic \& Siddha, New Delhi, India.

Lele Y, Thorve B, Tomar S, Parasnis A. 2017. Traditional Uses of The Wild Plants By The Tribal Communities Of Jawhar, Palghar, Maharashtra, India. International Journal of Botany and Research (IJBR) 7(6):19-22.

Macêdo MJ, Ribeiro DA, Santos MD, Macêdo DG, Macedo JG, Almeida BV, Saraiva ME, Lacerda MN, Souza MM. 2018. Fabaceae medicinal flora with therapeutic potential in Savanna areas in the Chapada do Araripe, Northeastern Brazil. Revista Brasileira de Farmacognosia. 28:738-750.

Mafuva C, Marima-Matarira HT. 2014. Towards professionalization of traditional medicine in Zimbabwe: a comparative analysis to the South African policy on traditional medicine and the Indian Ayurvedic system. International Journal of Herbal Medicine 2(2 Part C):154-161

Medicinal plants. http://envis.frlht.org/bot_search (Accessed 21/03/2019)

Nadkarni AK. 1999. Indian MateriaMedica, Vol- I. Bombay Popular Prakashan, Mumbai, India.

Natarajan B, Paulsen B. 2000. An Ethnopharmacological Study From Thane District, Maharashtra, India: Traditional Knowledge Compared With Modern Biological Science. Pharmaceutical Biology 38(2):139-151.

Nighnatu. http://niimh.nic.in/ebooks/e-Nighantu/(Accessed 11/09/2020)

Ouedraogo K, Dimobe K, Zerbo I, Etongo D, Zare A, Thiombiano A. 2019. Traditional knowledge and cultural importance of Gardenia erubescens Stapf\& Hutch. inSudanian savanna of Burkina Faso. Journal of Ethnobiology and Ethnomedicine 15:28.

Panda AK, Misra S. 2010. Health traditions of Sikkim Himalaya. Journal of Ayurveda and Integrative Medicine 1(3):183.

Pandit N. 2006. Raj Nighantu, with Dravyagunapra kashika hindi commentary, edited by Indradeva Tripathi, $4^{\text {th }}$ edition, Chowkhamba Krishnadas Academy, Varanasi.

Pardeshi K, Saokar R, Kadibagil VR, Nishakumari PR. 2016. Aushadhi Sevan Marga in Ayurevda, A Review. Unique Journal of Ayurvedic and Herbal Medicine 4(5):1-7.

Pasztory Z, Mohácsiné IR, Gorbacheva G, Börcsök Z. 2016. The utilization of tree bark. BioResources 11(3):7859-88.

Patil DA. 2006. Ethno botany of Nashik District Maharashtra. Daya publishing house, Delhi, India.

Pawar S, Patil DA. 2008. Ethnobotany of Jalgaon District of Maharashtra. Daya Publishing house, Delhi, India.

Phillips O, Gentry AH. 1993. The useful plants of Tambopata, Peru: I. Statistical hypotheses tests with a new quantitative technique. Economic Botany 47:15-32.

Press Information Bureau, Government of India. 2021. https://pib.gov.in/ (Accessed 01/08/2020)

Profile of Thane district. http://www.dcmsme.gov.in/publications/traderep/thane.htm (Accessed 13/07/2020) 
Rahman AH, Parvin MI. 2014. Study of medicinal uses on Fabaceae family at Rajshahi, Bangladesh. Research in Plant Sciences 23:2(1):6-8.

Rao NR, Henry AN. 1996. The Ethnobotany of Eastern Ghats, In Andhra Pradesh, India. Botanical Survey of India, India.

Rao RR. 2020. Floristic Diversity in Westren Ghats: Documentaion, Conservation and Bioprospection-A Priority agenda for action. Sahyadri E news. http://wgbis.ces.iisc.ernet.in/biodiversity/sahyadri_enews/ newsletter/issue38/article/index.htm (Accessed 09/09/2020)

Raunkiær C. 1934. The life forms of plants and statistical plant geography. Clarendon Press, Oxford, U.K.

Roy A, Janbandhu S. 2020. An ethnobotanical analysis on flora-medicine continuum among the tribal inhabitants of Ratnagiri and Palghar district, Maharashtra, India. Ethnobotany Research and Applications 20:1-23.

Sekhar AC, Reddi KR, Rajesh SS, Narendra K, Jangala S, Reddy PC, Sivaraman T. 2014. In vitro anti-venom potential of various Jatropha extracts on neutralizing cytotoxic effect induced by phospholipase A2 of crude venom from Indian cobra (Najanaja). Bangladesh Journal of Pharmacology 9(1):22-28.

Sethiya NK, Shekh MR, Singh PK. 2019. Wild banana [Ensetesuperbum (Roxb.) Cheesman.]: Ethnomedicinal, phytochemical and pharmacological overview. Journal of Ethnopharmacology 233:218-233.

Sharma PC, Yelne M B, Dennis TJ. 2001. Database on Medicinal plants used in Ayurveda Vol-2. Central Council For Research in Ayurvedic \& Siddha, New Delhi, India.

Sharma PC, Yelne M B, Dennis TJ. 2002. Database on Medicinal plants used in Ayurveda Vol-4. Central Council For Research in Ayurvedic \& Siddha, New Delhi, India.

Sharma PV. 2004. Classical uses of Medicinal plants. Chaukhambha Vishvabharati, Varanasi, India.

Sharma PV. 2013. DravyagunaVujnana. Vol II. ChaukhmabhaBharatiAcacdemy, Varanasi, India.

Sharma SK. 1998. Medicinal plants used in Ayurveda. Rashtriya Ayurveda Vidhyapith, New Delhi, India.

Sharngadhara. 2008. Sharngadhara Samhita, Gudharthadipika Sanskrit Commentary, edited by PanditParshuramShastri, 7th edition, Chaukhambha Orientalia, Varanasi.

Singh NP, Karthikeyan S. 2001. Flora of Maharashtra State (Flora of India Series 2): Dicotyledones. Botanical Survey of India, Calcutta, India.

Sree KS, Nagamalleswari K, Chaithra D, Yasodamma N. 2011. Traditional use of Calotropisprocera R. Br. against migraine. Current Science 101(9):1120-1121.

Suneetha J, Prasanthi S, Ramarao NB, Reddi TV. 2011. Indigenous phytotherapy for bone fractures from Eastern Ghats. Indian journal of Traditional Knowledge 10(3): 550-553.

Suntar I. 2019. Importance of ethnopharmacological studies in drug discovery: role of medicinal plants. Phytochemistry Reviews 1:1.

Surana V, Mishra SH, Satani B. 2016. Phytopharmacognostic Investigation and Evaluation of Antioxidant Properties of Leaves and Bark of HeterophragmaAdenophyllum. American Journal of Pharmaceutical and Technical Reseatch 6(4)

Sushrut. 2009. Sushruta Samhita with the 'Nibandhasangraha' commentary of Shri Dalhanacharya, edited by Vd. JadavajiTrikamji Acharya \&Narayanram Acharya. Chaukhambha Sanskrit Sansthan, Varanasi, India.

Tripurari K, ShrivastavaA, Kumar A, Laserson KF, Jai PN, Srinivasaraghavan V, Lakhbir SC, Francisco A. 2015. Viral Hepatitis Surveillance - India, 2011-2013. Morbidity and Mortality Weekly Report 64(28)758-762. Available on ,https://www.cdc.gov/Mmwr/preview/mmwrhtml/mm6428a3.htm (Accessed 15/05/2020)

Trivedi PC. 2002. Ethnobotany. Aavishkar Publishers, Jaipur, India.

Tugume P, Kakudidi EK, Buyinza M, Namaalwa J, Kamatenesi M, Mucunguzi P, Kalema J. 2016. Ethnobotanical survey of medicinal plant species used by communities around Mabira central Forest reserve, Uganda. Journal of Ethnobiology and Ethnomedicine 12(1):5. 
Uniyal MR. Tiwari RN. 1993. Medico-ethnobotany of Sonebhadra District, Utterpradesh. Central Council For Research in Ayurvedic\& siddha, New Delhi, India.

Uniyal SK, Singh KN, Jamwal P, Lal B. 2006. Traditional use of medicinal plants among the tribal communities of ChhotaBhangal, Western Himalaya. Journal of Ethnobiology and Ethnomedicine. 2(1):1-8.

Upadhye A, Kumbhojkar MS, Vartak VD. 1986. Observations on wild plants used in folk medicine in the rural areas of the Kolhapur district. Ancient Science of Life 6(2):119-121.

Vagbhat. 2002. AshtangaHridaya with the commentaries 'Sarvangasundara' of Arunadatta and 'Ayurvedarasayana' of Hemadri. Chowkhamba Sanskrit Sansthana, Varanasi, India.

Vaidya B. 1999. NighantuAadarsh. ChaukhmbhaVidyabhavan, Varanasi, India.

Vedavathy S, Sudhakar A, Mrdula V. 1997. Tribal medicinal plants of Chittoor. Ancient Science of Life 16(4):307-331

Vitalini S, Iriti M, Puricelli C, Ciuchi D, Segale A, Fico G. 2013. Traditional knowledge on medicinal and food plants used in Val San Giacomo (Sondrio, Italy) - An alpine ethnobotanical study. Journal of Ethnopharmacology 145(2):517-529.

Vulnerable tribal groups. http://krishi.maharashtra.gov.in/Site/Upload/Pdf/PoCRA_TPPF_new.pdf. (Accessed 29/08/2020)

Warrell DA, 1999. WHO/SEARO Guidelines for the clinical management of snakebite in the Southeast Asian Region, South East Asian Journal of Tropical Medicine and Public Health 30:1-85.

World Health Organisation. 2002. Traditional Medicine and Alternative Medicines. Geneva, Fact Sheet. No. 271.

World Health Organization (WHO). 2012. World Malaria Report. Available at: https://www.who.int/malaria/ publications/world_mala ria_report_2012/en/ (Accessed 16/05/2020) 